\title{
Boundaries of right-angled hyperbolic buildings
}

\author{
by \\ Jan Dymara (Wrocław) and Damian Osajda (Wrocław and Paris)
}

\begin{abstract}
We prove that the boundary of a right-angled hyperbolic building is a universal Menger space. As a consequence, the 3-dimensional universal Menger space is the boundary of some Gromov-hyperbolic group.
\end{abstract}

\section{INTRODUCTION}

Hyperbolic right-angled buildings were first explored by Marc Bourdon. The easiest non-trivial example can be glued from infinitely many pentagons. We glue them along edges - a finite number greater than 2 along each edge - so that a small neighbourhood of each vertex is a cone over a full bipartite graph. We want the obtained polyhedral complex to be connected and simply connected; this is easily arranged by passing to the universal cover of a connected component. A natural metric on our complex is a piecewise hyperbolic metric, each pentagon given the shape of a rightangled hyperbolic pentagon. This and similar examples were constructed and thoroughly investigated by Bourdon ([Bd1], [Bd2]) and Bourdon and Pajot ([BP1], [BP2]).

In particular, Bourdon states that the Gromov boundary of any of the complexes he considers is the Menger curve. There are two folklore proofs of this statement. The first follows the arguments of Benakli (cf. [Bd1], [B]). The second uses the result of Kapovich and Kleiner ([KK]): if the boundary of a one-ended hyperbolic group is 1-dimensional and has no local cut points then it is either the Menger curve or the Sierpiński carpet. This result applies to uniform lattices in the isometry groups of Bourdon's buildings. Since (as one can check) the boundary of a thick building contains a non-planar subset,

2000 Mathematics Subject Classification: 20E42, 54F35, 20F67.

Key words and phrases: hyperbolic building, Menger space, Gromov boundary.

Both authors were partially supported by KBN grants 2 P03A 01725 and N201 012 $32 / 0718$. The second author was a Marie Curie Intra-European fellow, contract MEIF CT 05011050 . 
it follows that in this case the boundary is the Menger curve. No details of either of the arguments have been published.

The purpose of this paper is to prove the following theorem.

MAIn TheORem. Let $X$ be a locally finite right-angled thick hyperbolic building of dimension $n \geq 2$. Then the Gromov boundary of $X$ is homeomorphic to the universal $(n-1)$-dimensional Menger space $\mu^{n-1}$.

A discussion of buildings explaining the meaning of our assumptions is contained in Section 1. Let us just mention that hyperbolic means Lobachevskiu-hyperbolic rather than Gromov-hyperbolic. The existence of $X$ as in the theorem is equivalent to the existence of a bounded finite right-angled polyhedron in $\mathbb{H}^{n}$. Therefore, $X$ exists only for $n=2,3,4$ ([Vin]). Once the polyhedron is given, $X$ can be constructed as the universal cover of some finite complex (cf. [D2], [GP] or Section 4). The fundamental group of this finite complex is quasi-isometric to $X$; thus, one gets examples of Gromovhyperbolic groups with Gromov boundary $\mu^{3}, \mu^{2}$ and $\mu^{1}$. The latter two spaces have been known to be boundaries of Gromov-hyperbolic groups (cf. $[\mathrm{BK}])$, but $\mu^{3}$ is new.

The proof of the main theorem is based on the following characterisation of $\mu^{n-1}$, due to Bestvina [Be]. A metric space $Y$ is homeomorphic to $\mu^{n-1}$ if and only if it is compact, $(n-1)$-dimensional, $(n-2)$-connected, locally $(n-2)$-connected, and has the $(n-1)$-dimensional disjoint disc property $\left(D D^{n-1} P\right)$. We check that these conditions are satisfied for the boundary $\partial X$ of an $n$-dimensional right-angled hyperbolic building $X$. In Lemma 3.1 we prove that $\partial X$ is compact and $(n-1)$-dimensional. Recall that $Y$ is locally $(n-2)$-connected if for each $y \in Y$ and every open neighbourhood $U$ of $y$ there exists an open set $V$ with $y \in V \subseteq U$ such that every map $S^{k} \rightarrow V$ extends to a map $B^{k+1} \rightarrow U$ (for $\left.k=0,1, \ldots, n-2\right)$. In Proposition 3.6 we check that $\partial X$ is $(n-2)$-connected and locally $(n-2)$-connected. The $(n-1)$-dimensional disjoint disc property says that for any two maps $f, g: D^{n-1} \rightarrow Y$ and any $\varepsilon>0$ there exist maps $f^{\prime}, g^{\prime}: D^{n-1} \rightarrow Y$ such that $f^{\prime}$ is $\varepsilon$-close to $f, g^{\prime}$ is $\varepsilon$-close to $g$, and $f^{\prime}\left(D^{n-1}\right) \cap g^{\prime}\left(D^{n-1}\right)=\emptyset$. A standard way to prove $D D^{n-1} P$ is to construct, for any $\varepsilon>0$, two maps $\phi, \psi: Y \rightarrow Y$, both $\varepsilon$-close to the identity map and with disjoint images. Such maps for $\partial X$ are constructed in Theorem 4.11 and Corollary 4.13 .

In Section 4, we investigate the structure of right-angled buildings, reproving some results of Globus ([Gl]) and Haglund and Paulin ([HP]). The advantages of our approach are as follows: (i) our assumptions on the thickness of the buildings are weaker; (ii) we obtain a deeper understanding of the automorphism group, allowing us to prove $D D^{n-1} P$.

Finally, in the appendix we prove that an $n$-dimensional hyperbolic (not necessarily right-angled) or Euclidean building is $(n-2)$-connected at infinity. 
This is a special case of some results of $[\mathrm{GP}]$ and $[\mathrm{DM}]$. We briefly criticise the arguments given in those papers (cf. $\left.\left[\mathrm{DM}^{\prime}\right]\right)$.

A variant of the main theorem has been proved independently by A. Dranishnikov and T. Januszkiewicz. We have not seen the details of their work.

We are grateful to Mike Davis, Frédéric Paulin, Tadeusz Januszkiewicz and Jacek Swiątkowski for helpful conversations.

\section{GENERALITIES ON BUILDINGS}

Two standard references for buildings are [Br] and [Ron]. Metrics on buildings are discussed in [D2], and hyperbolic buildings in [GP].

A Coxeter system is a pair $(W, S)$, where $W$ is a group, $S$ is a generating subset of $W$, and $W=\left\langle S \mid\left\{(s t)^{m_{s t}}\right\}_{s, t \in S}\right\rangle$. The numbers $m_{s t}$ are positive integers or infinity; $m_{s t}=1$ exactly when $s=t ; m_{s t}=\infty$ means that there is no relation between $s$ and $t$. We usually speak about a Coxeter group $W$, in fact meaning some Coxeter system $(W, S)$. A Coxeter group $W$ is right-angled if $m_{s t} \in\{1,2, \infty\}$ for all $s, t \in S$. A special subgroup of $W$ is a subgroup generated by some subset $T$ of $S: W_{T}=\langle T\rangle$. It is well known that $\left(W_{T}, T\right)$ is a Coxeter system. A subset $T \subset S$ is called spherical if $W_{T}$ is finite. For example, $\emptyset$ is spherical; $\{s\}$ is always spherical; $\{s, t\}$ is spherical unless $m_{s t}=\infty$. If $W$ is right-angled, then $T$ is spherical if and only if any two elements of $T$ commute. For $w \in W$ we define $\ell(w)$ to be the length of a shortest word in the generators $S$ representing $w$. We put

$$
\operatorname{In}(w)=\{s \in S \mid \ell(w s)<\ell(w)\} .
$$

It is well known that $\operatorname{In}(w)$ is always spherical.

Several different descriptions of buildings will be useful for us. We start with a combinatorial one. Let $W$ be a Coxeter group. We equip $W$ with a family $\left(\sim_{s}\right)_{s \in S}$ of equivalence relations, defined as follows: $w \sim_{s} v \Leftrightarrow$ $w \in\{v, v s\}$. Suppose that $A$ and $B$ are two sets, each equipped with an $S$ indexed family of equivalence relations. A map from $A$ to $B$ is a morphism if it preserves each of the equivalence relations; it is an isomorphism if it is a bijective morphism and if its inverse is also a morphism. A $W$-building is a set (of chambers) equipped with a family of equivalence relations $\left(\sim_{s}\right)_{s \in S}$ and with a family of subsets (called apartments) isomorphic to $W$, such that:

(B1) any two chambers are contained in some apartment;

(B2) if two chambers $x, y$ are both contained in apartments $A, A^{\prime}$, then there exists an isomorphism $A \rightarrow A^{\prime}$ fixing $x$ and $y$;

(B3) if apartments $A, A^{\prime}$ contain a chamber $x$ and both intersect an equivalence class $R$ of one of the relations $\sim_{s}$, then there exists an isomorphism $A \rightarrow A^{\prime}$ fixing $x$ and mapping $R \cap A$ to $R \cap A^{\prime}$. 
For the equivalence of this definition and a more standard one see [Ron, Thm 3.11]. A building is called thick if each equivalence class of each relation $\sim_{s}$ has at least three elements. We will call a building locally finite if each equivalence class of each relation $\sim_{s}$ is finite. For example, $W$ is a locally finite building, but it is not thick. Chambers $x, y$ such that $x \sim_{s} y$ are called $s$-adjacent or simply adjacent. A gallery in a building $X$ is a sequence of chambers such that any two consecutive elements are adjacent. A finite gallery is minimal if there is no shorter gallery with the same extremities. For a subset $T \subseteq S$ and $x \in X$ we define the residue $\operatorname{Res}(x, T)$ as the set of all $y \in X$ such that there exists a gallery of the form $x=x_{0} \sim_{s_{1}} x_{1} \sim$ $\cdots \sim_{s_{k}} x_{k}=y$, where $s_{1}, \ldots, s_{k} \in T$. For $X=W$ the $T$-residue of $x$ is the left $W_{T}$-coset containing $x: \operatorname{Res}(x, T)=x W_{T}$. In general, it is well known that $\operatorname{Res}(x, T)$ is a $W_{T}$-building.

The notion of folding map is very important for us. Let $X$ be a $W$ building. Pick any chamber $B \in X$. By (B1), for any $x \in X$ there exists an apartment $A$ such that $B, x \in A$. Let $\iota_{A}: A \rightarrow W$ be the unique isomorphism which sends $B$ to 1 . Then, by (B2), $\iota_{A}(x)$ does not depend on $A$. The formula $\pi_{B}(x)=\iota_{A}(x)$ defines the (B-based) folding map $\pi_{B}: X \rightarrow W$. This map is a morphism of buildings (one uses (B3) to check that). We often abbreviate $\pi_{B}$ to $\pi$. Here is a list of some well-known and useful properties of $\pi$.

(F1) If $x \in X$ and $t \in \operatorname{In}(\pi(x))$, then there exists a unique $x^{t} \in X$ such that $x \sim_{t} x^{t}$ and $\pi\left(x^{t}\right)=\pi(x) t$.

(F2) The image under $\pi$ of a minimal gallery in $X$ starting at $B$ is a minimal gallery in $W$. Conversely, if $x \in X$ then any minimal gallery in $W$ from $B$ to $\pi(x)$ is the image under $\pi$ of a unique minimal gallery from $B$ to $x$.

For $x \in X$ we may define its length (meaning the distance from $B$ ) in terms of the folding: $\ell(x)=\ell(\pi(x))$.

(F3) For any $x \in X$ and $T \subseteq S$ there exists a unique shortest chamber $y$ in $\operatorname{Res}(x, T)$. Moreover, if $z \in \operatorname{Res}(x, T)$, then there exists a minimal gallery from $B$ to $z$ via $y$. The restriction of $\pi$ to $\operatorname{Res}(x, T)$ composed with left multiplication by $\pi(y)^{-1}$ coincides with the $y$-based folding $\operatorname{map} \pi_{y}: \operatorname{Res}(x, T) \rightarrow W_{T}$.

Buildings also have geometric realisations. The most general construction is due to Davis. Let $D$ be a topological space with a family $\left(D_{s}\right)_{s \in S}$ of subspaces ( $D$ is a model for a chamber; $D_{s}$ is a model for the intersection of two $s$-adjacent chambers). For $p \in D$ we put $S(p)=\left\{s \in S \mid p \in D_{s}\right\}$. Now for any $W$-building $X$ Davis defines $X_{D}=X \times D / \sim$, where $(x, p) \sim(y, q) \Leftrightarrow$ $p=q$ and $x \in \operatorname{Res}(y, S(p))$. The best choice for $D$ is the Davis chamber $K$ : it is defined as the geometric realisation of the poset of all spherical subsets 
of $S$ (including $\emptyset$ ); the subspace $K_{s}$ is the subcomplex spanned by subsets containing $\{s\}$. We will denote $X_{K}$ by $|X|$. For $X=W$ one obtains the Davis complex $|W|$. The geometric realisation $|Y|$ of a subset $Y$ of $X$ is the subset of $|X|$ given by $|Y|=\left\{[(y, p)]_{\sim} \mid y \in Y, p \in K\right\}$, where $[(y, p)]_{\sim}$ denotes the $\sim$-equivalence class of $(y, p)$. Apartments in $|X|$ are geometric realisations of apartments in $X$. The folding map induces a map $|\pi|:|X| \rightarrow|W|$, which is also called the folding map and is usually denoted by $\pi$. Here are some nice properties of $|X|$ :

- if $X$ is locally finite, then $|X|$ is locally compact;

- $|X|$ is contractible;

- $|X|$ carries a piecewise-Euclidean $\mathrm{CAT}(0)$ metric (the Moussong metric, cf. $[\mathrm{M}])$.

Let $P$ be a convex polytope in the hyperbolic space $\mathbb{H}^{n}$. Suppose that each dihedral angle of $P$ is of the form $\pi / k$, where the positive integer $k$ may vary from angle to angle. Then the reflections in codimension-one faces of $P$ generate a Coxeter group $W$; Coxeter groups arising in this way will be called hyperbolic. The group $W$ acts on $\mathbb{H}^{n}$ with fundamental domain $P$ (this is a theorem of Poincaré). The barycentric subdivision of $P$ is isomorphic to the Davis chamber $K$ corresponding to the group $W$. Using this isomorphism one can define a polyhedral structure and a piecewise hyperbolic metric on $|X|$ for any $W$-building $X$. Then each apartment in $|X|$ is isometric to $\mathbb{H}^{n}$, with chambers corresponding to $W$-translates of $P$ in $\mathbb{H}^{n}$. Moreover, the whole building $|X|$ is $\mathrm{CAT}(-1)$ (cf. [D2], [GP]). A building $X$ (often meaning the geometric realisation $|X|$, equipped with the CAT $(-1)$ metric and the polyhedral structure described above) corresponding to a hyperbolic Coxeter group will be called a hyperbolic building. If all dihedral angles of $P$ are $\pi / 2$, then $P, W$ and $X$ are called right-angled.

The Gromov boundary $\partial X$ of a hyperbolic building $X$ (or, more generally, of a $\mathrm{CAT}(-1)$ space, cf. $[\mathrm{BH}])$ can be defined as the set of geodesic rays $\gamma:[0, \infty) \rightarrow X$ starting at some fixed point $x_{0}$. The topology on $\partial X$ is defined by the basis of open sets $\left\{U_{r}(x) \mid x \in X, r>0\right\}$, where

$$
U_{r}(x)=\left\{\gamma \in \partial X \mid \gamma([0, \infty)) \cap B_{r}(x) \neq \emptyset\right\}
$$

$\left(B_{r}(x)\right.$ is the open ball in $X$ of radius $r$ centred at $\left.x\right)$. The topological space thus obtained is independent of the choice of $x_{0}$. We will always choose $x_{0}$ in the interior of a chamber. For $p, q \in X \cup \partial X$ we denote by $\overline{p q}$ the geodesic segment from $p$ to $q$ (which exists and is unique because $X$ is a $\operatorname{CAT}(-1)$ space). We define the topology on $X \cup \partial X$ by the basis of open sets consisting of open balls in $X$ and sets

$$
V_{r}(x)=\left\{y \in X \cup \partial X \mid \overline{x_{0} y} \cap B_{r}(x) \neq \emptyset\right\} ;
$$


restricted to $\partial X$, this topology yields the topology described above. If $X$ is locally compact then $X \cup \partial X$ is a compactification of $X$. The folding map $\pi: X \rightarrow \mathbb{H}^{n}$ extends to a map $\pi: X \cup \partial X \rightarrow \mathbb{H}^{n} \cup \partial \mathbb{H}^{n}$, where $\partial \mathbb{H}^{n}$ is the Gromov boundary of the hyperbolic space.

\section{HALF-SPACES}

The purpose of this section is to prove some auxiliary facts about buildings. In Subsection 2. A we give a different basis of open sets for the topology on $X \cup \partial X$; in 2.B we prove some properties of the elements of this new basis; in 2.C we discuss connectedness properties of some subsets of spherical buildings.

2.A. Standard neighbourhoods. In this subsection we assume that $X$ is a hyperbolic building. We keep the notation $\left(W, P, \pi, x_{0}, B_{r}(x), V_{r}(x)\right)$ as in the final two paragraphs of Section 1. In particular, $\pi$ is the folding map based at a chamber $B$ and sending $B$ to $P$. We choose $x_{0}$ in the interior of $B$. We denote by $p_{R}$ the geodesic retraction of $X \cup \partial X$ onto $\overline{B_{R}\left(x_{0}\right)}: p_{R}(x)$ is the intersection point of $S_{R}\left(x_{0}\right)$ and $\overline{x_{0} x}$ if $d\left(x_{0}, x\right)>R$; otherwise $p_{R}(x)=x$. We also use $p_{R}$ to denote the corresponding retraction in $\mathbb{H}^{n} \cup \partial \mathbb{H}^{n}$.

Let $H$ be a hyperplane in $\mathbb{H}^{n}=|W|$ containing a codimension-one face of some $W$-translate $w P$ of $P$. Such hyperplane is called a wall and divides $\mathbb{H}^{n}$ into two open connected pieces: $H^{+}$and $H^{-}$(our convention is $\left.\operatorname{int}(P) \subseteq H^{-}\right)$. We put $\partial H^{+}=\left\{y \in \partial \mathbb{H}^{n} \mid \overline{p y} \cap H^{+} \neq \emptyset\right\}$, for some $p \in \operatorname{int}(P)$ (the result does not depend on the choice of $p$; one may choose $\left.p=\pi\left(x_{0}\right)\right)$. Let $\mathcal{H}$ be the set of all connected components of sets of the form $\pi^{-1}\left(H^{+} \cup \partial H^{+}\right)$, over all walls $H$. Since all $B_{r}(x)$ and $V_{r}(x)$ are pathwise connected (any $y \in V_{r}(x)$ can be connected by a part of $\overline{x_{0} y}$ to a point in $B_{r}(x)$ ), the space $X \cup \partial X$ is locally pathwise connected. Therefore, elements of $\mathcal{H}$ are open; they will be used as neighbourhoods of boundary points, and called standard (open) neighbourhoods. By convention, the whole space $X \cup \partial X$ is also a standard open neighbourhood. We claim that $\mathcal{H} \cup\left\{B_{r}(x) \mid x \in X, r>0\right\}$ is another basis of open sets for the topology on $X \cup \partial X$. This is implied by the following lemma.

Lemma 2.1. Let $x \in \partial X$ and $U \subset X \cup \partial X$ be its neighbourhood. Then there exists a wall $H$ such that one of the connected components of the set $\pi^{-1}\left(H^{+} \cup \partial H^{+}\right)$contains $x$ and is contained in $U$.

Proof. By the definition of the topology on $X \cup \partial X$, and because $x_{0} \in$ $\operatorname{int}(B)$, one can find a point $x_{1}$ lying on the geodesic ray $\overline{x_{0} x}$ and inside a chamber $C$, and a positive number $r$, such that $B_{r}\left(x_{1}\right) \subseteq \operatorname{int}(C)$ and $V_{r}\left(x_{1}\right) \subseteq U$. Then $V_{r}\left(x_{1}\right)$ is an open neighbourhood of $x$ contained in $U$. 
Claim. There exists a wall $F \subset \mathbb{H}^{n}$ such that

$$
\pi(x) \in F^{+} \cup \partial F^{+} \subset \pi\left(V_{r}\left(x_{1}\right)\right) .
$$

Proof. Notice that $\pi\left(V_{r}\left(x_{1}\right)\right)$ equals $V_{r}\left(\pi\left(x_{1}\right), \mathbb{H}^{n}\right)$, hence is an open neighbourhood of $\pi(x)$ in $\mathbb{H}^{n} \cup \partial \mathbb{H}^{n}$. Indeed, this follows easily from the following two observations: $\pi\left(B_{r}\left(x_{1}\right)\right)=B_{r}\left(\pi\left(x_{1}\right), \mathbb{H}^{n}\right)$; any geodesic segment in $X$ starting at $x_{0}$ is mapped by $\pi$ onto a geodesic segment in $\mathbb{H}^{n}$.

Choose $R>0$ such that every hyperplane in $\mathbb{H}^{n}$ contained in $\mathbb{H}^{n} \backslash$ $B_{R}\left(\pi\left(x_{0}\right), \mathbb{H}^{n}\right)$ and intersecting the geodesic ray $\overline{\pi\left(x_{0}\right) \pi(x)}$ is contained in $\pi\left(V_{r}\left(x_{1}\right)\right)$. Only finitely many walls intersect $B_{R}\left(\pi\left(x_{0}\right), \mathbb{H}^{n}\right)$, while infinitely many walls intersect $\overline{\pi\left(x_{0}\right) \pi(x)}$; therefore, there exists a wall $F$ intersecting $\overline{\pi\left(x_{0}\right) \pi(x)}$ and contained in $\mathbb{H}^{n} \backslash B_{R}\left(\pi\left(x_{0}\right), \mathbb{H}^{n}\right)$. By the choice of $R$, this $F$ is contained in $\pi\left(V_{r}\left(x_{1}\right)\right)$, and satisfies the conditions of the claim. aclaim

Let now $F$ be as in the claim. Let $\widetilde{F}^{+}$be the connected component of $\pi^{-1}\left(F^{+} \cup \partial F^{+}\right)$containing $x$. Let $D$ be the length of a geodesic segment $\overline{\pi\left(x_{0}\right) q}$ which is tangent to $S_{r}\left(\pi\left(x_{1}\right), \mathbb{H}^{n}\right)$ at $q$. Then we have $\pi\left(p_{D}\left(\widetilde{F}^{+}\right)\right) \subseteq$ $p_{D}\left(F^{+} \cup \partial F^{+}\right) \subset B_{r}\left(\pi\left(x_{1}\right), \mathbb{H}^{n}\right)$, hence $p_{D}\left(\widetilde{F}^{+}\right) \subset \pi^{-1}\left(B_{r}\left(\pi\left(x_{1}\right), \mathbb{H}^{n}\right)\right)$. Recall that $B_{r}\left(x_{1}\right)$ is contained in the interior of one chamber. Therefore, $B_{r}\left(x_{1}\right)$ is one of the connected components of $\pi^{-1}\left(B_{r}\left(\pi\left(x_{1}\right), \mathbb{H}^{n}\right)\right)$. However, $\widetilde{F}^{+}$is connected and its closure contains $x$, hence $p_{D}\left(\widetilde{F}^{+}\right)$is connected and contains $p_{D}(x)$. Since $p_{D}(x)=p_{D}\left(x_{1}\right)$, we have $p_{D}\left(\widetilde{F}^{+}\right) \subset B_{r}\left(x_{1}\right)$. This implies $\widetilde{F}^{+} \subset V_{r}\left(x_{1}\right) \subset U$.

-Lemma 2.1

Lemma 2.2. Let $H$ be a wall, and let $x \in \pi^{-1}\left(H^{+}\right)$. Then the connected component of $x$ in $\pi^{-1}\left(H^{+}\right)$is dense in the connected component of $x$ in $\pi^{-1}\left(H^{+} \cup \partial H^{+}\right)$.

Proof. Since $X \cup \partial X$ is locally pathwise connected, connected components of open sets in this space are pathwise connected. Let $y$ be in the connected component of $x$ in $\pi^{-1}\left(H^{+} \cup \partial H^{+}\right)$, and let $\gamma:[0,1] \rightarrow X \cup \partial X$ be a path from $x$ to $y$ contained in that component. We may choose $R$ so large that $p_{R} \circ \gamma$ is a path starting at $x$ and contained in $\pi^{-1}\left(H^{+}\right)$. Concatenating this path with $\overline{p_{R}(y) y}$ we obtain a path from $x$ to $y$ which is contained in $\pi^{-1}\left(H^{+}\right)$, except perhaps for its endpoint $y$. It follows that $y$ belongs to the closure of the component of $x$ in $\pi^{-1}\left(H^{+}\right)$.

2.B. Shortest elements. In this subsection $W$ is a right-angled Coxeter group (we assume that $W$ is hyperbolic only in Propositions 2.12 and 2.13). Our goal is to prove that any half-space in $W$ has a unique shortest element (Prop. 2.5); we will also investigate the corresponding question for buildings (Prop. 2.11). A half-space in $W$ is a set of the form

$$
H(w, s)=\{h \in W \mid d(h, w s)<d(h, w)\},
$$


where $w \in W, s \in S$ and $d\left(w_{1}, w_{2}\right)=\ell\left(w_{1}^{-1} w_{2}\right)$. The name is motivated by the fact that if $W$ is hyperbolic then the geometric realisation of $H(w, s)$ is a closed half-space in the usual sense in $|W|=\mathbb{H}^{n}$.

We begin with two principles which are very useful when dealing with distances in Coxeter groups.

$( \pm 1) d(a s, b)=d(a, b) \pm 1$ and $d(a, b s)=d(a, b) \pm 1$, for every $a, b \in W$ and every $s \in S$.

(R) Let $t, t^{\prime} \in S$ be two distinct commuting generators of $W$, let $R$ be a $\left\{t, t^{\prime}\right\}$-residue in $W$, and let $x \in W$. Then the four distances from $x$ to chambers of $R$ yield three consecutive integers, the middle one attained twice, on two non-adjacent chambers of $R$.

Property (R) follows from properties $( \pm 1)$ and (F3) (the latter is stated in Section 1). Now we proceed to some preliminary lemmas. The proofs are quite standard, so we omit the details.

Lemma 2.3. Suppose $h \in H(w, s) \backslash\{w s\}, h t \notin H(w, s)$ for some $t \in S$. Then there exists $t^{\prime} \in S$ such that $t^{\prime} t=t t^{\prime}$ and $d\left(h t^{\prime}, w s\right)<d(h, w s)$. Moreover:

(a) $h t^{\prime} \in H(w, s), h t^{\prime} t \notin H(w, s)$;

(b) $H(h t, t)=H\left(h t t^{\prime}, t\right)$.

Proof. Choose $t^{\prime}$ such that $h t^{\prime}$ is closer to $w s$ than $h$. Using $( \pm 1)$ one can deduce that $h t^{\prime}$ is then closer to $w$ than $h$. Therefore, $t, t^{\prime} \in \operatorname{In}\left(w^{-1} h\right)$, hence $t t^{\prime}=t^{\prime} t$. It remains to prove (a) and (b).

We apply property $(\mathrm{R})$ to the residue $R=\operatorname{Res}\left(h,\left\{t, t^{\prime}\right\}\right)$. First, we take $x=w$ and $x=w s$. The eight distances are easily determined up to a common additive constant; part (a) follows. Second, take an arbitrary $x \in W$. Then there are four cases to consider, depending on which element of $R$ is closest to $x$. In each case it is readily checked that $x \in H(h t, t)$ if and only if $x \in H\left(h t t^{\prime}, t\right)$; this proves (b).

Lemma 2.4. Suppose $h \in H(w, s), h t \notin H(w, s)$ for some $t \in S$. Then $t=s$ and $H(h s, s)=H(w, s)$.

Proof. Take a counterexample (to the claim $t=s$ ) which is closest to $w$. Lemma 2.3 produces a counterexample which is even closer to $w$, a contradiction. The same argument proves the second claim.

Lemma 2.5. Suppose that $h \in H(w, s)$ and $h s \notin H(w, s)$. Then there exists a minimal gallery $w s, w_{s t}, \ldots$, wst $_{1} \ldots t_{m}=h$ such that $t_{i} s=s t_{i}$. The converse is also true.

Proof. The first statement follows from Lemma 2.3. The converse is easily proved by induction on $m$ : one should apply property $(\mathrm{R})$ to $R=$ $\operatorname{Res}\left(w s t_{1} \ldots t_{m},\left\{t_{m}, s\right\}\right)$ and $x=w, w s$. 
Corollary 2.6.

(a) The set $\{h \in W \mid h \in H(w, s)$, hs $\notin H(w, s)\}$ coincides with ws $W_{\{s\}^{\prime}}$, where $\{s\}^{\prime}=\{t \in S \backslash\{s\} \mid t s=s t\}$.

(b) Any half-space is gallery connected.

Proof. Part (a) follows directly from Lemma 2.5. To prove (b), consider a gallery from $x \in H(w, s)$ to $w$. Let $y$ be the first element of that gallery which does not belong to $H(w, s)$. Then, by Lemma 2.4 and part (a), ys $\in w s W_{\{s\}^{\prime}}$, so that it can be connected to $w s$ by a gallery in $w s W_{\{s\}}$. Concatenating the part from $x$ to $y s$ of the first gallery with the second gallery we obtain a gallery in $H(w, s)$ connecting $x$ to $w s$. Statement (b) follows.

Proposition 2.7. Every half-space (in any right-angled Coxeter group) has a unique shortest element.

Proof. We assume that 1 does not belong to our half-space $H(w, s)$ otherwise the statement is trivial. Let $x \in H(w, s)$, and let $y$ be the first element in a minimal gallery from $x$ to 1 which does not belong to $H(w, s)$. Then, by Lemma 2.4 and Corollary 2.6, ys $\in w s W_{\{s\}^{\prime}}$. Any residue contains a unique shortest element; let $g$ be the shortest element in $w s W_{\{s\}^{\prime}}$. There exists a minimal gallery from $y s$ via $g$ to 1 (cf. property (F3), Section 1). Consequently, $\ell(x) \geq \ell(y s) \geq \ell(g)$; equalities hold only if $x=y=g$. It follows that $g$ is the unique shortest element in $H(w, s)$.

The proof of Proposition 2.7 has the following corollary.

Corollary 2.8. Any element of $H(w, s)$ can be connected with 1 by a minimal gallery passing through the shortest element of $H(w, s)$.

Proof. In the situation of the proof of Proposition 2.7, concatenate the part from $x$ to $y s$ of the first gallery with the second gallery. The result is a minimal gallery from $x$ via $g$ to 1 .

We now turn to buildings. Let $X$ be a $W$-building, and let $\pi: X \rightarrow W$ be the $B$-based folding map. We also fix a half-space $H(w, s)$. We assume that $1 \notin H(w, s)$ (because we are eventually interested in standard open neighbourhoods) and that $w s$ is the shortest element of $H(w, s)$ (we may do so because of Lemma 2.4).

Lemma 2.9. Suppose that $x \in X, \pi(x) \in H(w, s)$. Then there exists a minimal gallery $\left(x_{0}=x, x_{1}, \ldots, x_{\ell}=B\right)$ such that $\pi\left(x_{k}\right)=w s$ for $k=$ $d(\pi(x), w s)$. If $\left(x_{0}^{\prime}=x_{0}, x_{1}^{\prime}, \ldots, x_{\ell}^{\prime}\right)$ is another such gallery, then $x_{k}^{\prime}=x_{k}$.

Proof. Let $\pi(x)=g$. If $\sigma=\left(g, g s_{1}, \ldots, g s_{1} \ldots s_{\ell}\right)$ is a minimal gallery from $g$ to 1 via $w s=g s_{1} \ldots s_{k}$, then $\widetilde{\sigma}=\left(x, x^{s_{1}},\left(x^{s_{1}}\right)^{s_{2}}, \ldots\right)$ is the unique gallery from $x$ to $B$ that folds onto $\sigma$ (cf. properties (F1) and (F2) of the folding map). Moreover, $\widetilde{\sigma}$ is minimal. This gives the first assertion. 
Now let $\tau=\left(g=\pi\left(x_{0}^{\prime}\right), \pi\left(x_{1}^{\prime}\right), \ldots, \pi\left(x_{\ell}^{\prime}\right)=1\right)$. By Tits' solution of the word problem in Coxeter groups, the gallery $\sigma$ can be transformed into $\tau$ by a sequence of moves of the form

$$
\begin{gathered}
\eta=\left(\ldots, h_{i}, h_{i+1}=h t, h_{i+2}=h t u, \ldots\right) \\
\downarrow \\
\xi=\left(\ldots, h_{i}^{\prime}=h_{i}, h_{i+1}^{\prime}=h u, h_{i+2}^{\prime}=h u t=h t u=h_{i+2}, \ldots\right)
\end{gathered}
$$

where $t u=u t$. Moreover, this can be done with the $k$ th chamber of the gallery equal to $w s$ throughout the process (just operate separately on the gallery segments from $g$ to $w s$ and from $w s$ to 1$)$. Let $\left(\sigma=\sigma_{1}, \ldots, \sigma_{m}=\tau\right)$ be a sequence of galleries corresponding to such a transformation. Notice that in the situation of the move $(*)$ the galleries $\widetilde{\eta}, \widetilde{\xi}$ coincide except for the $(i+1)$ st chamber. This is because both $\left(y^{u}\right)^{t}$ and $\left(y^{t}\right)^{u}$ are the shortest element in $\operatorname{Res}(y,\{t, u\})$ so that they coincide (here $y$ denotes the common $i$ th element of $\widetilde{\eta}$ and $\widetilde{\xi}$ ). It follows that the $k$ th chamber of each $\widetilde{\sigma}_{i}$ is the same; therefore $x_{k}=x_{k}^{\prime}$.

We now define combinatorial and geometric counterparts of standard open neighbourhoods, in the setting of general right-angled buildings. Let $x \in X$ and $\pi(x) \in H(w, s)$. We define $Y \subseteq X$ as follows: $y \in Y$ if there exists a gallery $\left(x=x_{0} \sim_{s_{1}} x_{1} \sim_{s_{2}} \cdots \sim_{s_{m}} x_{m}=y\right)$ such that $\operatorname{Res}\left(\pi\left(x_{i}\right), s_{i}\right) \subseteq$ $H(w, s)$. We also define

$$
\begin{aligned}
H(w, s)_{r} & =\{[g, p] \in|W| \mid \operatorname{Res}(g, S(p)) \subseteq H(w, s)\}, \\
Y_{r} & =\pi^{-1}\left(H(w, s)_{r}\right) \cap|Y| \\
& =\{[y, p] \in|X| \mid y \in Y, \operatorname{Res}(\pi(y), S(p)) \subseteq H(w, s)\} .
\end{aligned}
$$

Notice that $Y=\left\{y \in X \mid \operatorname{int}(|y|) \subseteq Y_{r}\right\}$.

LEMMA 2.10. $Y_{r}$ is pathwise connected and both closed and open in $\pi^{-1}\left(H(w, s)_{r}\right)$.

Proof. 1) $Y_{r}$ is pathwise connected: Recall that the Davis chamber $K$ is the geometric realisation of the poset of all spherical subsets of $S$. The vertex of $K$ corresponding to $\emptyset$ will be denoted $\operatorname{bar}(K)$, and the vertex corresponding to $\{s\}$ by $\operatorname{bar}\left(K_{s}\right)$. The corresponding points in a chamber $|z| \subseteq|X|$ will be denoted $\operatorname{bar}(|z|)$, bar $\left(|z|_{s}\right)$. Any point in $|z|$ can be connected to bar $(|z|)$ by a line segment contained in $|z|$.

Let $[y, p] \in Y_{r}$; the segment from $[y, p]$ to $\operatorname{bar}(|y|)$ is contained in $Y_{r}$. Now let $\left(x=x_{0} \sim_{s_{1}} x_{1} \sim_{s_{2}} \cdots \sim_{s_{m}} x_{m}=y\right)$ be a gallery as in the definition of $Y$. The piecewise linear path $\operatorname{bar}\left(\left|x_{0}\right|\right)-\operatorname{bar}\left(\left|x_{1}\right|_{s_{1}}\right)-\operatorname{bar}\left(\left|x_{1}\right|\right)-\cdots-\operatorname{bar}(|y|)$ is contained in $Y_{r}$ : the only problematic points are $p_{i}=\operatorname{bar}\left(\left|x_{i}\right|_{s_{i}}\right)$; however, $S\left(p_{i}\right)=\left\{s_{i}\right\}$ and $\operatorname{Res}\left(\pi\left(x_{i}\right), s_{i}\right) \subseteq H(w, s)$. Thus, any point in $Y_{r}$ can be connected by a path to $\operatorname{bar}(|x|)$ so that $Y_{r}$ is pathwise connected. 
2) $Y_{r}$ is open: Let $[y, p] \in Y_{r}$. Let $V$ be the subset of the Davis chamber $K$ consisting of points $q$ such that $S(q) \subseteq S(p)$. Then $\bigcup_{z \in \operatorname{Res}(y, S(p))} z \times V$ is an open subset of $X \times K$, closed under the equivalence relation defining $|X|$. Therefore $\bigcup_{z \in \operatorname{Res}(y, S(p))}\{[z, v] \mid v \in V\}$ is an open neighbourhood of $[y, p]$. This neighbourhood is contained in $Y_{r}$.

3) $Y_{r}$ is closed in $\pi^{-1}\left(H(w, s)_{r}\right)$ : Let $[z, q]$ be in the closure of $Y_{r}$ in $|X|$. Let $N$ be the open neighbourhood of $[z, q]$ constructed in 2); then $N \cap Y_{r}$ $\neq \emptyset$. Let $[y, p] \in Y_{r} \cap N$. Since $[y, p]$ is in the closure of the interior of $|y|$, some interior points of $|y|$ belong to $N$. This implies that $y \in \operatorname{Res}(z, S(q))$, and $[z, q]=[y, q]$. We are done, unless $\operatorname{Res}(\pi(y), S(q))$ is not contained in $H(w, s)$; in that case, though, $[\pi(z), q]=[\pi(y), q] \notin H(w, s)_{r}$ and $[z, q] \notin$ $\pi^{-1}\left(H(w, s)_{r}\right)$.

\section{Proposition 2.11. $Y$ has a unique shortest chamber.}

Proof. Let $\sigma$ be a minimal gallery from $y \in Y$ to $B$ via $\pi^{-1}(w s)$; we denote by $a(y)$ the element of $\sigma$ that folds onto $w s$ (this element is well defined due to Lemma 2.9).

Suppose now that $y, y^{\prime} \in Y, y \sim_{t} y^{\prime}$ and $\operatorname{Res}(\pi(y), t) \subseteq H(w, s)$. We will prove that $a(y)=a\left(y^{\prime}\right)$. If $y^{\prime}=y^{t}$, then there exists a gallery $\sigma$ from $y$ to $B$ via $a(y)$ passing through $y^{\prime}$ (see the construction of a gallery in the proof of Corollary 2.8: one can start constructing $\sigma$ by shortening $y$ in an arbitrary manner, provided one does not leave $H(w, s)$ ), hence $a(y)=a\left(y^{\prime}\right)$ in this case. The case $y=y^{\prime t}$ is analogous. Now suppose that the shortest element $u$ of $\operatorname{Res}(y, t)$ is different from both $y$ and $y^{\prime}$. Since $y \in Y$ and $\operatorname{Res}(\pi(u), t)=\operatorname{Res}(\pi(y), t) \subseteq H(w, s)$ we have $u \in Y$. Then $u=y^{t}=y^{\prime t}$, and $a(y)=a(u)=a\left(y^{\prime}\right)$ by the previous case.

It follows that if $y \in Y$ and $\left(x=x_{0}, \ldots, x_{l}=y\right)$ is a gallery as in the definition of $Y$, then $a(y)=a\left(x_{l-1}\right)=\cdots=a(x)$. Consequently, any $y \in Y \backslash\{a(x)\}$ is strictly longer than $a(y)=a(x)$. Thus $a(x)$ is the unique shortest element of $Y$.

We conclude with two propositions summarising the above discussion in the hyperbolic case.

Proposition 2.12. Suppose that $W$ is a right-angled hyperbolic Coxeter group, associated to a polyhedron $P \subseteq \mathbb{H}^{n}$, and let $H$ be a wall. Then among all $w \in W$ such that $\operatorname{int}(w P) \subseteq H^{+}$there is a unique shortest one; let us call it $w_{0}$. Suppose that $H$ contains the face $w_{0} P_{s}$ of $P$; then, for any $x_{0} \in \operatorname{int}(P)$, the geodesic through $x_{0}$ perpendicular to $H$ intersects $H$ in the interior of the face $w_{0} P_{s}$.

Proof. Suppose that $H$ contains the face $w P_{s}$ of the chamber $w P$. We may assume that $\ell(w)<\ell(w s)$ (swapping $w$ and $w s$ if necessary). Then, under the usual identification of $|W|$ and $\mathbb{H}^{n}$, the geometric realisation of 
$H(w, s)$ corresponds to the closed half-space $\overline{H^{+}}$. This follows easily from the fact that the distance between two elements of $W$ is equal to the number of walls separating the corresponding chambers. Now the first assertion of the proposition follows from Proposition 2.7.

For the second assertion observe that the geodesic $\gamma$ passing through $x_{0}$ and perpendicular to $H$ intersects $H$ at an interior point $\gamma(t)$ of the face $w P_{s}=w s P_{s}$. Indeed, otherwise $\gamma(t) \in H^{\prime}$ for some wall $H^{\prime} \perp H$; then, however, the image of $\gamma$ is contained in $H^{\prime}$, and cannot contain $x_{0}$. Now if $w s \neq w_{0}$, then there exists a wall $H^{\prime} \perp H$ separating $w s P$ from $w_{0} P$ (and hence from $\left.x_{0}\right)$. Since $\gamma \perp H, \gamma$ does not intersect $H^{\prime}$. On the other hand, $\gamma$ connects points $x_{0}$ and $\gamma(t)$ lying on different sides of $H^{\prime}$, a contradiction.

Proposition 2.13. Suppose that $W$ is a right-angled hyperbolic Coxeter group, associated to a polyhedron $P \subseteq \mathbb{H}^{n}$, and let $H$ be a wall. Let $w_{0}$ be the element of $W$ defined in Proposition 2.12. Suppose further that $X$ is a $W$-building, and that $U$ is a connected component of $\pi^{-1}\left(H^{+} \cup \partial H^{+}\right)$. Then $\pi^{-1}\left(w_{0} P\right) \cap U$ consists of one chamber.

Proof. Choose $w \in W$ and $s \in S$ such that $H$ is the wall separating $w P$ from $w s P$. We use the notation introduced before Lemma 2.10; we choose $x$ such that $\operatorname{int}(|x|) \subseteq U$. Note that $H(w, s)_{r}=H^{+}$. By Lemma 2.10, $Y_{r}$ is the connected component of $\pi^{-1}\left(H^{+}\right)$that contains int $(|x|)$. Then Lemma 2.2 implies that $Y_{r}$ is also equal to the intersection of $U$ and $|X|$. Recall that $Y=\left\{y \in X \mid \operatorname{int}(|y|) \subseteq Y_{r}\right\}$. Therefore, the proposition follows from Proposition 2.11.

2.C. Halves and quarters of spherical buildings. In this subsection, $Y$ is a finite right-angled $W$-building. Such buildings are spherical, in the following sense. Let $\Delta$ be a simplex of dimension $|S|-1$, and let $\Delta_{s}$ be distinct codimension-one faces of $\Delta$, for $s \in S$ (the Davis chamber of $W$ would be isomorphic to a cone over the first barycentric subdivision of $\Delta$ ). Then the apartments in $Y_{\Delta}$ are triangulated spheres. One equips $W_{\Delta}=S^{n-1}$ with the standard CAT(1) metric, in such a way that each simplex of the triangulation is isometric to a right-angled spherical simplex. Then one pulls this metric back by a folding map to a piecewise spherical metric on $Y_{\Delta}$. Thus one obtains the standard CAT(1) metric on $Y_{\Delta}$. In this subsection we abbreviate $Y_{\Delta}$ to $Y$.

Buildings as above appear as small spheres around vertices (or, more generally, as small normal spheres of cells) in right-angled hyperbolic buildings. When dealing with complements of balls in a hyperbolic building or in a standard open neighbourhood it is natural to consider certain subsets of spherical buildings. In this subsection we define such subsets and prove their higher connectedness in the right-angled case. 
Let $B \in Y$ be a chamber, and let $\pi: Y \rightarrow S^{n-1}$ be the $B$-based folding map. We choose $\pi$ so as to have $\pi(B)=\left\{\left(x_{i}\right) \in S^{n-1} \mid x_{1}, \ldots, x_{n} \leq 0\right\}$. Then $\pi$ is a simplicial map for the following triangulation of $S^{n-1}$ : any simplex $\sigma \subseteq S^{n-1}$ is defined by a conjunction of $n$ conditions of the form $x_{i} \leq 0$, $x_{i}=0, x_{i} \geq 0$, one for each $i$. Let $C$ be the $(n-1)$-simplex in $S^{n-1}$ which is antipodal to $\pi(B)$, i.e., $C=\left\{\left(x_{i}\right) \in S^{n-1} \mid x_{1}, \ldots, x_{n} \geq 0\right\}$. We choose any $v \in \operatorname{int}(\pi(B))$; in other words, $v$ is a unit vector in $\mathbb{R}^{n+1}$ with all coordinates negative. We denote by $E^{+}$the hemisphere $\left\{x \in S^{n-1} \mid\langle x, v\rangle \leq 0\right\}$, and we put $Y^{+}=\pi^{-1}\left(E^{+}\right)$.

\section{Lemma 2.14. $\pi^{-1}(C)$ is a deformation retract of $Y^{+}$.}

Proof. We first construct a deformation retraction $r_{t}$ from $E^{+}$to $C$. The idea is as follows. If $e \in C$ then put $r_{t}(e)=e$. If $e \notin C \cup \pi(B)$ then there exists a minimal simplex in our triangulation of $S^{n-1}$ containing $e$; this simplex is a join of some face of $\pi(B)$ and some face of $C$. There exists a unique great circle containing $e$ and intersecting those two faces; our retraction moves $e$ along that circle towards $C$. In other words, let $e=e_{-}+e_{+}$, where $\left(e_{-}\right)_{i}=\min \left(e_{i}, 0\right)$ and $\left(e_{+}\right)_{i}=\max \left(e_{i}, 0\right)$. We put

$$
r_{t}(e)=t e_{-}+\sqrt{t^{2}+\frac{1-t^{2}}{\left|e_{+}\right|^{2}}} e_{+} ;
$$

notice that this expression is continuous in $(t, e) \in[0,1] \times\left(S^{n-1} \backslash \pi(B)\right)$. We have

$$
\frac{d}{d t}\left\langle v, r_{t}(e)\right\rangle=\left\langle e_{-}, v\right\rangle+\frac{t}{\sqrt{t^{2}+\frac{1-t^{2}}{\left|e_{+}\right|^{2}}}}\left(1-\frac{1}{\left|e_{+}\right|^{2}}\right)\left\langle e_{+}, v\right\rangle .
$$

This expression is non-negative for $e \in S^{n-1} \backslash \pi(B)$, therefore $e \in E^{+}$implies $r_{t}(e) \in E^{+}$. For $x \in Y^{+}$we define $R_{t}(x)$ as follows: choose any simplex $\Sigma$ of $Y$, containing $x$; then $R_{t}(x) \in \Sigma, \pi\left(R_{t}(x)\right)=r_{t}(\pi(x))$. Note that if $e \in E^{+}$ and $e \in \sigma$ for some face $\sigma$ of $S^{n-1}$, then $r_{t}(e) \in \sigma$; therefore the definition of $R_{t}(x)$ does not depend on the choice of $\Sigma$.

It is well known (a self-contained proof is found in Section 4.1) that a finite right-angled building is a join. More specifically, let $Y_{i}=\{x \in Y \mid$ $\left.(\forall j \neq i)\left(\pi(x)_{j}=0\right)\right\}$. Then $Y$ is isomorphic as a simplicial complex and homeomorphic as a topological space to the join of the sets $Y_{i}, i=1, \ldots, n$. Observe that $\pi^{-1}(C)$ is isomorphic to the join of the sets $Y_{i} \backslash B$. It follows that $\pi^{-1}(C)$ is $(n-2)$-connected (being a join, it is a finite building, and thus has the homotopy type of a bouquet of $(n-1)$-spheres). Now Lemma 2.14 implies the following.

LEMma 2.15. $Y^{+}$is $(n-2)$-connected. 
Now put $Y_{i}^{+}=\left\{x \in Y^{+} \mid \pi(x)_{i} \geq 0\right\}$. It is clear that $Y_{i}^{+}$is $R_{t}$-invariant so it retracts to $\pi^{-1}(C)$. In particular, we get as before:

LEMMA 2.16. $Y_{i}^{+}$is $(n-2)$-connected.

\section{LOCAL CONNECTEDNESS}

In this section we prove higher connectedness (local and global) of the boundary of a hyperbolic building. The general strategy is Morse-theoretic, à la $[\mathrm{BB}]$ and $[\mathrm{BCM}]$. Let us fix our notational conventions.

- $W$ denotes a right-angled hyperbolic Coxeter group acting on $\mathbb{H}^{n}$ with fundamental domain $P$.

- $X$ is a locally finite $W$-building (meaning the Davis realisation with the $\mathrm{CAT}(-1)$ metric).

- $B$ is some fixed chamber of $X$ (to be called the base chamber).

- $\pi=\pi_{B}: X \rightarrow \mathbb{H}^{n}$ is the $B$-based folding map such that $\pi(B)=P$.

- $x_{0}$ is some fixed generic point in the interior of $B$ (the genericity conditions will be specified later).

- $S_{R}(x, Y)$ and $B_{R}(x, Y)$ are the sphere and the open ball of radius $R$ and centre $x$ in a metric space $Y$. If $Y=X$, then we use the abbreviations $S_{R}(x)$ and $B_{R}(x)$. If, additionally, $x=x_{0}$, then we write simply $S_{R}$ and $B_{R}$.

- $p_{R}: X \cup \partial X \rightarrow X$ is the geodesic retraction onto $\bar{B}_{R}$, i.e., $p_{R}(x)$ is the intersection point of $\overline{x x_{0}}$ and $S_{R}$ if $d\left(x, x_{0}\right) \geq R$, and $p_{R}(x)=x$ otherwise.

LEMMA 3.1. $\partial X$ is an $(n-1)$-dimensional compactum.

Proof. Consider an inverse system $\left\{\left(S_{k}, p_{k}\right)\right\}_{k=1}^{\infty}$ of spheres centred at $x_{0}$ with $p_{k}: S_{k+1} \rightarrow S_{k}$ being the geodesic projections onto $S_{k}$. Then $\partial X=$ inv $\lim \left\{\left(S_{k}, p_{k}\right)\right\}$. As every $S_{k}$ is an $(n-1)$-dimensional compactum, $\partial X$ is an at most $(n-1)$-dimensional compactum. But since it contains $S^{n-1}$ (the boundary of an apartment isometric to $\mathbb{H}^{n}$ ) it has dimension $n-1$.

LEMMA 3.2. Let $U$ be a standard neighbourhood of a point of $\partial X$ and let $R>d\left(x_{0}, U\right)$. Then $\bar{U} \cap S_{R}$ is a deformation retract of $\bar{U} \backslash B_{R}$.

Proof. Roughly speaking, the retraction is executed by the gradient flow of the restriction of the function $d\left(x_{0}, \cdot\right)$ to $\bar{U} \backslash B_{R}$. The case $U=X$ is easy: the retraction is $\left(p_{t}\right)_{t \in[R,+\infty]}$, where $p_{+\infty}=\operatorname{Id}_{X}$.

Let $U$ be a connected component of $\pi^{-1}\left(H^{+} \cup \partial H^{+}\right)$, for some wall $H \subseteq \mathbb{H}^{n}$. We identify $\mathbb{H}^{n}$ with the Poincaré disc $\mathbb{D}^{n}$ in such a way that $\pi\left(x_{0}\right)$ corresponds to 0 . Then let $Z(x)=-x$ be the vector field on $\mathbb{D}^{n}$ pointing towards 0 . We define a vector field $V$ on $\overline{H^{+}} \backslash B_{R}\left(\pi\left(x_{0}\right), \mathbb{H}^{n}\right)$ as follows. If $x \in H^{+} \cup \partial H^{+} \cup \partial H$ then we put $V(x)=Z(x)$. If $x \in H$ then 
$V(x)$ is proportional to the orthogonal projection of $Z(x)$ onto $T_{x} H$; the proportionality constant is chosen so that the radial component of $V(x)$ is equal to $Z(x)$. The vector field $V$ is not continuous; nevertheless, it defines a continuous flow $\varphi_{V}^{t}$. The trajectory $\varphi_{V}^{t}(x)$ follows the geodesic $\overline{x \pi\left(x_{0}\right)}$ until it hits $H$; then it moves inside $H$ along a geodesic towards the projection of $\pi\left(x_{0}\right)$ onto $H$. The trajectory stops when it hits $S_{R}\left(\pi\left(x_{0}\right), \mathbb{H}^{n}\right)$ (this may happen before it reaches $H)$. Observe that if a trajectory intersects some wall $H^{\prime} \neq H$, then it moves from $\left(H^{\prime}\right)^{+}$to $\left(H^{\prime}\right)^{-}$. Therefore, the flow $\varphi_{V}^{t}$ lifts to a flow $\psi_{V}^{t}$ on $\bar{U} \backslash B_{R}$. This lift defines a retraction of $\bar{U} \backslash B_{R}$ onto $\bar{U} \cap S_{R}$.

LEMma 3.3. Let $U$ be a standard neighbourhood of a point of $\partial X$. Then $\bar{U} \cap S_{R}$ is $(n-2)$-connected for every $R>0$.

Proof. Let $U$ be a component of $\pi^{-1}\left(H^{+} \cup \partial H^{+}\right)$for some wall $H$. (The case $U=X \cup \partial X$ is very similar.) It follows from Propositions 2.12 and 2.13 that for $t$ slightly greater than $d\left(\pi\left(x_{0}\right), H\right)$ the intersection $\bar{U} \cap S_{t}$ is contained in a single chamber. This intersection is then a disc, hence is contractible.

Next we would like to understand how the topology of $\bar{U} \cap S_{t}$ changes as $t$ grows. The picture is somewhat reminiscent of Morse theory: the topology changes only at some critical radii. Suppose that $S_{t}\left(\pi\left(x_{0}\right), \mathbb{H}^{n}\right)$ intersects a (closed) face $\sigma \subseteq H \cup H^{+}$of our polyhedral structure at some point $p \in \sigma$. We say that the intersection is critical if $\sigma$ is perpendicular to $\overline{\pi\left(x_{0}\right) p}$ at $p$; $t$ is then called a critical radius. We make a generic choice of $x_{0}$ to ensure that critical intersections occur only at interior points of the corresponding faces $(p \in \operatorname{int}(\sigma))$, and that to each critical $t$ there corresponds a unique critical intersection. Notice that $\sigma$ can be a vertex of our polyhedral structure. Let $d\left(\pi\left(x_{0}\right), H\right)=t_{0}<t_{1}<t_{2}<\cdots$ be the sequence of all critical radii. It is clear that for $t, t^{\prime} \in\left(t_{i}, t_{i+1}\right)$ the spaces $S_{t} \cap \bar{U}$ and $S_{t^{\prime}} \cap \bar{U}$ are homeomorphic (cf. [BCM]). We will show that for every $i$ and every sufficiently small positive $\varepsilon$ the space $S_{t_{i}+\varepsilon} \cap \bar{U}$ is $(n-2)$-connected provided $S_{t_{i}-\varepsilon} \cap \bar{U}$ is $(n-2)$ connected.

We will first deal with the simplest case: the face $\sigma$ corresponding to $t_{i}$ is a vertex $p \in H^{+}$. Let $\operatorname{Res}(p)$ be the union of all faces in $\mathbb{H}^{n}$ which contain $p$. We choose $\delta>0$ such that the sphere $D:=S_{\delta}\left(p, \mathbb{H}^{n}\right)$ is contained in $\operatorname{int}(\operatorname{Res}(p))$. Let $H_{p}$ be the hyperplane passing through $p$ and orthogonal to $\overline{\pi\left(x_{0}\right) p}$. This hyperplane divides $D$ into two hemispheres, $D^{-}$ (the one closer to $\left.\pi\left(x_{0}\right)\right)$ and $D^{+}$. There exists an $\varepsilon \in(0, \delta)$ such that $D \cap H_{p}=D \cap S_{t_{i}+\varepsilon}\left(\pi\left(x_{0}\right), \mathbb{H}^{n}\right)$; if necessary, we decrease $\delta$ so as to have $\varepsilon<\min \left\{t_{i+1}-t_{i}, t_{i}-t_{i-1}\right\}$. The sphere $D$ inherits a triangulation from the polyhedral structure on $\mathbb{H}^{n}$. We want $S_{t_{i}+\varepsilon}\left(\pi\left(x_{0}\right), \mathbb{H}^{n}\right)$ and $S_{t_{i}-\varepsilon}\left(\pi\left(x_{0}\right), \mathbb{H}^{n}\right)$ to intersect this triangulation "in the same way". More precisely, we require that there be a homeomorphism of $D$ mapping each simplex into itself and 
transforming $D \cap S_{t_{i}+\varepsilon}\left(\pi\left(x_{0}\right), \mathbb{H}^{n}\right)$ into $D \cap S_{t_{i}-\varepsilon}\left(\pi\left(x_{0}\right), \mathbb{H}^{n}\right)$. This condition can be achieved by further decreasing $\delta$ (and consequently $\varepsilon$ ).

Next we pass to the building. By Lemma 3.2, $\bar{U} \cap S_{t_{i} \pm \varepsilon}$ is homotopy equivalent to $\bar{U} \backslash B_{t_{i} \pm \varepsilon}$. Let $\pi^{-1}(p)=\left\{p_{1}, \ldots, p_{k}\right\}$, and let $D_{j}=\pi^{-1}(D) \cap \overline{B_{\delta}\left(p_{j}\right)}$, $D_{j}^{+}=\pi^{-1}\left(D^{+}\right) \cap \overline{B_{\delta}\left(p_{j}\right)}$. We have $D_{j}^{+}=D_{j} \backslash B_{t_{i}+\varepsilon}$. Put $K_{j}=\overline{B_{\delta}\left(p_{j}\right)}$, and let $Y^{+}$be the closure of $\left(\bar{U} \backslash B_{t_{i}+\varepsilon}\right) \backslash \bigcup_{j=1}^{k} K_{j}$. Furthermore, let $Y_{j}^{+}=$ $Y^{+} \cup K_{1} \cup \cdots \cup K_{j}$ for $j=0,1, \ldots, k$. We will prove, by downward induction on $j$, that $Y_{j}^{+}$is $(n-2)$-connected. The space $Y_{k}^{+}$is homotopy equivalent to $\bar{U} \backslash B_{t_{i}-\varepsilon}$ (here we need the condition that $S_{t_{i} \pm \varepsilon}\left(\pi\left(x_{0}\right), \mathbb{H}^{n}\right)$ intersect $D$ "in the same way"), hence it is $(n-2)$-connected; $(n-2)$-connectedness of $Y^{+}$ will imply the same property for the homotopy equivalent space $\bar{U} \backslash B_{t_{i}+\varepsilon}$. Observe that the sets $K_{j}$ are pairwise disjoint, and that $Y_{j}^{+}$is obtained from $Y_{j-1}^{+}$by gluing $K_{j}$ along $D_{j}^{+}$. By Lemma $2.15, D_{j}^{+}$is $(n-2)$-connected, while $K_{j}$ is clearly contractible. Therefore:

1. Connectedness of $Y_{j}^{+}$implies that of $Y_{j-1}^{+}$.

2. $(n>2)$ By van Kampen's theorem,

$$
\pi_{1}\left(Y_{j}^{+}\right)=\pi_{1}\left(Y_{j-1}^{+}\right) *_{\pi_{1}\left(D_{j}^{+}\right)} \pi_{1}\left(K_{j}\right) .
$$

Since $\pi_{1}\left(K_{j}\right)=\pi_{1}\left(D_{j}^{+}\right)=0$ this implies $\pi_{1}\left(Y_{j-1}^{+}\right)=\pi_{1}\left(Y_{j}^{+}\right)=0$.

3. $(n>3)$ From the Mayer-Vietoris sequence

$$
\cdots \rightarrow H_{l}\left(D_{j}^{+}\right) \rightarrow H_{l}\left(K_{j}\right) \oplus H_{l}\left(Y_{j-1}^{+}\right) \rightarrow H_{l}\left(Y_{j}^{+}\right) \rightarrow H_{l-1}\left(D_{j}^{+}\right) \rightarrow \cdots
$$

we get $H_{l}\left(Y_{j-1}^{+}\right)=H_{l}\left(Y_{j}^{+}\right)$for $l \leq n-2$.

The conclusion now follows from the Hurewicz theorem.

Now we discuss the general case: $t_{i}$ is a critical radius, $\sigma$ the corresponding face, $p$ the intersection point of $S_{t_{i}}\left(\pi\left(x_{0}\right), \mathbb{H}^{n}\right)$ and $\sigma$. We choose $\delta$ so that $S_{\delta}\left(p, \mathbb{H}^{n}\right) \subseteq \operatorname{int}(\operatorname{Res}(p))$, and we choose $\varepsilon$ so that $S_{\delta}\left(p, \mathbb{H}^{n}\right) \cap H_{p}=S_{\delta}\left(p, \mathbb{H}^{n}\right) \cap$ $S_{t_{i}+\varepsilon}\left(\pi\left(x_{0}\right), \mathbb{H}^{n}\right)$. Let $\sigma_{p}^{\perp}$ be the maximal hyperplane orthogonal to $\sigma$ at $p$. We put $D=\sigma_{p}^{\perp} \cap S_{\delta}\left(p, \mathbb{H}^{n}\right) \cap \overline{H^{+}}$(intersecting with $\overline{H^{+}}$is only necessary if $p \in H)$ and $D^{+}=D \backslash B_{t_{i}+\varepsilon}\left(\pi\left(x_{0}\right), \mathbb{H}^{n}\right)$. Again, by decreasing $\delta$ we ensure that $\varepsilon<\min \left\{t_{i+1}-t_{i}, t_{i}-t_{i-1}\right\}$ and that the spheres $S_{t_{i} \pm \varepsilon}\left(\pi\left(x_{0}\right), \mathbb{H}^{n}\right)$ intersect $D$ "in the same way". We also set $K=\overline{B_{\delta}\left(p, \mathbb{H}^{n}\right)} \cap \overline{H^{+}}$and $L=$ $\sigma \cap S_{\delta}\left(p, \mathbb{H}^{n}\right)$.

We pass to the building. Let $\pi^{-1}(p)=\left\{p_{1}, \ldots, p_{k}\right\}$. We have chosen $\delta$ so small that $\pi^{-1}\left(\overline{B_{\delta}\left(p, \mathbb{H}^{n}\right)}\right)$ is the disjoint union of the $\overline{B_{\delta}\left(p_{j}\right)}$. We put $D_{j}=\pi^{-1}(D) \cap \overline{B_{\delta}\left(p_{j}\right)}, D_{j}^{+}=\pi^{-1}\left(D^{+}\right) \cap \overline{B_{\delta}\left(p_{j}\right)}, K_{j}=\pi^{-1}(K) \cap \overline{B_{\delta}\left(p_{j}\right)}$, $L_{j}=\pi^{-1}(L) \cap \overline{B_{\delta}\left(p_{j}\right)}$. Then we define $Y^{+}$and $Y_{j}^{+}$exactly as before. Notice that $K_{j}$ is homeomorphic to a cone over the join $L_{j} * D_{j}$, and is attached to $Y_{j-1}^{+}$along a subset of the base of that cone homeomorphic to $L_{j} * D_{j}^{+}$. By 
Lemma 2.15 (if $p \in H^{+}$) or by Lemma 2.16 (if $p \in H$ ), $D_{j}^{+}$is $(n-d-2$ )connected, where $d=\operatorname{dim}(\sigma)$. Then $L_{j} * D_{j}^{+}$is $(n-2)$-connected by the suspension theorem $\left(L_{j}\right.$ is a $(d-1)$-dimensional sphere). Moreover, $K_{j}$ is contractible. In the remaining part of the argument (1.-3.) we just replace $D_{j}^{+}$by (a homeomorphic copy of) $L_{j} * D_{j}^{+}$.

LEMma 3.4. Let $U$ be a standard neighbourhood of $x \in \partial X$ in $X \cup \partial X$. Then for every standard neighbourhood $V$ of $x$ whose closure is contained in $U$ and every map $f:\{0,1\} \rightarrow V \cap \partial X$ there exists an extension $g: I=$ $[0,1] \rightarrow U \cap \partial X$ of $f$.

Proof. We will construct the desired map $g$ as the limit of a sequence $\left(g_{i}\right)_{i=0}^{\infty}$ of maps $g_{i}: I \rightarrow S_{N_{i}} \cap U$, for an increasing sequence of integers $N_{i}$.

Assume that we have defined a natural number $N_{i}$, a map $g_{i}: I \rightarrow$ $S_{N_{i}} \cap U$, and additionally finite families $V_{i}$ and $U_{i}$ of standard open neighbourhoods of points of $\partial X$, and a triangulation $\mathcal{T}_{i}$ of $I$ of mesh at most $2^{-i}$ together with a map $h_{i}:\left|\mathcal{T}_{i}^{(0)}\right| \rightarrow U \cap \partial X$ and a map $s_{i}: \mathcal{T}_{i}^{(1)} \rightarrow U_{i}$. (Note that by $\mathcal{T}^{(j)}$ we denote the set of $j$-simplices of a triangulation $\mathcal{T}$, and $\left|\mathcal{T}^{(j)}\right|$ denotes a geometric realization of the $j$-skeleton of $\mathcal{T}$.) Assume that they satisfy the following conditions:

(i) $\left.h_{i}\right|_{\{0,1\}}=f$,

(ii) $\left.g_{i}\right|_{\left|\mathcal{T}_{i}^{(0)}\right|}=p_{N_{i}} \circ h_{i}$,

(iii) $\left(\forall \tau \in \mathcal{T}_{i}^{(1)}\right)\left(\exists B \in V_{i}\right)\left(\bar{B} \subset s_{i}(\tau), g_{i}(\partial \tau) \subset B\right.$ and $\left.g_{i}(\tau) \subset \bar{B}\right)$,

(iv) $\left(\forall B \in V_{i}\right) \bar{B} \subset U$.

We will show how to find a natural $N_{i+1}$, a map $g_{i+1}$ etc. For every $D \in V_{i}$ one can find finite families $U_{i+1}^{D}$ and $V_{i+1}^{D}$ of standard open neighbourhoods of points of $\partial X$ such that

(a) $\bar{D} \cap \partial X \subset \bigcup V_{i+1}^{D}$,

(b) $\left(\forall C \in U_{i+1}^{D}\right)\left(\forall A \in U_{i}\right)$ if $\bar{D} \subset A$ then $\bar{C} \subset A$,

(c) $\left(\forall A \in U_{i+1}^{D}\right) \bar{A} \subset U \backslash B_{N_{i}}$,

(d) $\left(\forall B \in V_{i+1}^{D}\right)\left(\exists A \in U_{i+1}^{D}\right) \bar{B} \subset A$.

Define finite families $V_{i+1}$ and $U_{i+1}$ by $V_{i+1}=\bigcup_{D \in V_{i}} V_{i+1}^{D}$ and $U_{i+1}=$ $\bigcup_{D \in V_{i}} U_{i+1}^{D}$. Find a natural $N_{i+1}>N_{i}$ such that for every $D \in V_{i}$ we have $S_{N_{i+1}} \cap \bar{D} \subset \bigcup V_{i+1}^{D}$. Given a 1-simplex $\tau$ of $\mathcal{T}_{i}$, by (iii) we find $D_{\tau} \in V_{i}$ with $\bar{D}_{\tau} \subset s_{i}(\tau), g_{i}(\partial \tau) \subset D_{\tau}$ and $g_{i}(\tau) \subset \bar{D}_{\tau}$. Every standard open neighbourhood $D$ has the following property: for any $R>0$ and any $y \in X \cup \partial X$, if $p_{R}(y) \in D$ then $y \in D$. Observe that $p_{N_{i}}\left(p_{N_{i+1}} \circ h_{i}(\partial \tau)\right)=g_{i}(\partial \tau) \subset D_{\tau}$; therefore $\left.p_{N_{i+1}} \circ h_{i}\right|_{\partial \tau}$ maps $\partial \tau$ into $S_{N_{i+1}} \cap D_{\tau}$. By Lemma 3.3 we can extend this map to $g_{i+1}^{\tau}: \tau \rightarrow S_{N_{i+1}} \cap \bar{D}_{\tau}$. Define $g_{i+1}$ as the union of $g_{i+1}^{\tau}$ over all $\tau \in \mathcal{T}_{i}^{(1)}$. By continuity of $g_{i+1}$ one can choose a subdivision $\mathcal{T}_{i+1}$ of the 
triangulation $\mathcal{T}_{i}$ of $I$ with simplices of diameter at most $2^{-i-1}$, so fine that for every 1-simplex $\sigma$ of $\mathcal{T}_{i+1}$ contained in a 1-simplex $\tau$ of $\mathcal{T}_{i}$ there exists $B \in V_{i+1}^{D_{\tau}}$ such that $g_{i+1}^{\tau}(\sigma) \subset B$. Then, by (d), for any $\sigma, \tau$ and $B$ as in the previous sentence there exists an $s_{i+1}(\sigma) \in U_{i+1}^{D_{\tau}}$ satisfying $\bar{B} \subset s_{i+1}(\sigma)$. Observe that, by (b), $\overline{s_{i+1}(\sigma)} \subset s_{i}(\tau)$. Finally, we define $h_{i+1}$ as follows: for $v \in \mathcal{T}_{i}^{(0)}$ we put $h_{i+1}(v)=h_{i}(v)$; for $v \in \mathcal{T}_{i+1}^{(0)} \backslash \mathcal{T}_{i}^{(0)}$ we choose any point $h_{i+1}(v) \in \partial X$ such that $p_{N_{i+1}}\left(h_{i+1}(v)\right)=g_{i+1}(v)$.

To start the construction of $g_{i}$ 's one has to define: $N_{0}, g_{0}, V_{0}$ and $U_{0}, \mathcal{T}_{0}$, $h_{0}$ and $s_{0}$. Let $N_{0}$ be a natural number such that $S_{N_{0}} \cap V \supset p_{N_{0}} \circ f\left(S^{0}\right)$. By Lemma 3.3 one can find a map $g_{0}: I \rightarrow S_{N_{0}} \cap \bar{V}$ extending the map $p_{N_{0}} \circ f:\{0,1\} \rightarrow S_{N_{0}}$. Then set $V_{0}=\{V\}, U_{0}=\{U\}, \mathcal{T}_{0}$ the triangulation of $B^{1}$ consisting of one 1-simplex, $h_{0}=f$ and $s_{0}(v)=U$ for every $v \in \mathcal{T}_{0}^{(0)}$. Then it is obvious that conditions (i)-(iv) are satisfied.

We will now show some properties of the sequence $\left(g_{i}\right)_{i=1}^{\infty}$ of maps which will imply that its limit is a continuous map extending $f$.

Claim 0. $g_{i}(\tau) \subset s_{L}(\tau)$ for $\tau \in \mathcal{T}_{L}^{(1)}$ and $i \geq L$.

Proof. First we show that for $i, j=0,1,2, \ldots$ and for any two simplices $\sigma \in \mathcal{T}_{i}^{(1)}$ and $\varrho \in \mathcal{T}_{i+j}^{(1)}$ such that $\varrho \subset \sigma$ we have $s_{i+j}(\varrho) \subset s_{i}(\sigma)$. We proceed by induction on $j$. For $j=0$ the inclusion is obvious, and for $j=1$ it follows from the construction of $s_{i}$. Assume we have proved that $s_{i+j}(\varrho) \subset$ $s_{i}(\sigma)$. Let $\kappa \in \mathcal{T}_{i+j+1}^{(1)}$ be a simplex contained in a simplex $\varrho \in \mathcal{T}_{i+j}^{(1)}$ that is itself contained in $\sigma \in \mathcal{T}_{i}^{(1)}$. Then, by the induction assumptions, we have $s_{i+j+1}(\kappa) \subset s_{i+j}(\varrho) \subset s_{i}(\sigma)$. This finishes the induction.

Let $A=\left\{\sigma \in \mathcal{T}_{i}^{(1)} \mid \sigma \subset \tau\right\}$. Then $\tau=\bigcup_{\sigma \in A} \sigma$, and

$$
g_{i}(\tau)=g_{i}\left(\bigcup_{\sigma \in A} \sigma\right)=\bigcup_{\sigma \in A} g_{i}(\sigma) \subset \bigcup_{\sigma \in A} s_{i}(\sigma) \subset s_{L}(\tau) .
$$

Here the last inclusion follows from what we proved above, and the first one holds by (iii). Claim 0

Claim 1. For every $y \in I$ the limit $\lim _{i \rightarrow \infty} g_{i}(y)$ exists.

Proof. Take an arbitrary open (in $X \cup \partial X$ ) finite cover $\mathcal{W}$ of $\bar{U} \cap \partial X$. For every $j \geq i>0$ and every $A \in U_{j}$ we have $A \subset U \backslash B_{N_{i-1}}$. Therefore there exists a natural $L>0$ such that for every $i \geq L$ every neighbourhood $A \in U_{i}$ is contained in some member of $\mathcal{W}$. Take an arbitrary $y \in I$. Let $\tau \in \mathcal{T}_{L}$ be a maximal simplex containing $y$. Then, by Claim $0, g_{i}(\tau) \subset s_{L}(\tau) \subset W$ for every $i \geq L$ and some $W \in \mathcal{W}$. This implies the existence of the limit. 
Claim 2. $\lim _{i \rightarrow \infty} g_{i}(y) \in U \cap \partial X$.

Proof. This follows from: $g_{i}(y) \in \bigcup U_{1}$ for every $i ; \bar{A} \subset U$ for every $A \in U_{1}$. Claim 2

Claim 3. The formula $g(x)=\lim _{i \rightarrow \infty} g_{i}(x)$ defines a continuous map $g: I \rightarrow U \cap \partial X$.

Proof. As in the proof of Claim 1, for every finite open cover $\mathcal{W}$ there exists $L>0$ such that for every $i \geq L$ and any $A \in U_{i}$ the star $\bigcup \operatorname{St}(A)$ of $A$ in $U_{i}$ is contained in some member of $\mathcal{W}$. Take an arbitrary $y \in I$. Let $\tau \in \mathcal{T}_{L}$ be a maximal simplex containing $y$. As in Claim 1 , we have $g_{i}(\sigma) \subset s_{L}(\sigma)$ for every $i \geq L$ and every 1 -simplex $\sigma$ of $\mathcal{T}_{L}$ which has non-empty intersection with $\tau$; hence, $g_{i}(\bigcup \operatorname{St}(\tau)) \subset \bigcup\left\{s_{L}(\sigma) \mid \sigma \in \operatorname{St}(\tau)\right\} \subset \bigcup \operatorname{St}\left(s_{L}(\tau)\right) \subset W$ for some $W \in \mathcal{W}$. In other words, for every open cover $\mathcal{W}$ as above and any given $y \in I$ there exists a natural $L, W \in \mathcal{W}$, and an open neighbourhood $E \subset I$ of $y$ such that for every $i \geq L$ we have $g_{i}(E) \subset W$. This implies that the limit of $g_{i}$ 's is continuous. Claim 3

Claim 4. The map $g: I \rightarrow U \cap \partial X$ extends $f$.

Proof. This follows from the fact that $\left.g_{i}\right|_{\{0,1\}}=\left.p_{N_{i}} \circ h_{i}\right|_{\{0,1\}}=p_{N_{i}} \circ f$ and $\lim _{i \rightarrow \infty} p_{N_{i}} \circ f(y)=f(y)$ for every $y \in\{0,1\}$. Claim 4 Lemma 3.4

Lemma 3.5. Let $U$ be a standard neighbourhood of $x \in \partial X$ in $X \cup \partial X$. Then for every standard neighbourhood $V$ of $x$ whose closure is contained in $U$, every $k \in\{0,1, \ldots, n-2\}$ and every map $f: S^{k} \rightarrow V \cap \partial X$ there exists an extension $g: B^{k+1} \rightarrow U \cap \partial X$ of $f$.

Proof. We will proceed by induction on $k$.

1. The case of $k=0$ was proved in Lemma 3.4 above.

2. Induction step. Assume we have proved the lemma for $k=0,1, \ldots$, $M-1$. Let $V$ and $f: S^{M} \rightarrow V \cup \partial X$ be given. Again, we will construct the desired $g: B^{M+1} \rightarrow U \cap \partial X$ as the limit of a sequence $\left(g_{i}\right)_{i=0}^{\infty}$ of maps $g_{i}: B^{M+1} \rightarrow S_{N_{i}} \cap U$, where $N_{i}$ is an increasing sequence of integers.

Assume that we have defined a natural number $N_{i}$, a map $g_{i}: B^{M+1} \rightarrow$ $S_{N_{i}} \cap U$, finite families $V_{i}^{1}$ and $U_{i}^{1}$ of standard open neighbourhoods of points of $\partial X$, and a triangulation $\mathcal{T}_{i}$ of $B^{M+1}$ of mesh at most $2^{-i}$ together with a map $h_{i}:\left|\mathcal{T}_{i}^{(M)}\right| \rightarrow U \cap \partial X$ and a map $s_{i}: \mathcal{T}_{i}^{(M+1)} \rightarrow U_{i}^{1}$. Assume that they satisfy the following conditions:

(i) $\left.h_{i}\right|_{S^{M}}=f$,

(ii) $\left.g_{i}\right|_{\left|\mathcal{T}_{i}^{(M)}\right|}=p_{N_{i}} \circ h_{i}$

(iii) $\left(\forall \tau \in \mathcal{T}_{i}^{(M+1)}\right)\left(\exists B \in V_{i}^{1}\right)\left(\bar{B} \subset s_{i}(\tau), g_{i}(\partial \tau) \subset B\right.$ and $\left.g_{i}(\tau) \subset \bar{B}\right)$,

(iv) $\left(\forall B \in V_{i}^{1}\right) \bar{B} \subset U$. 
We will show how to find a natural $N_{i+1}$, a map $g_{i+1}$ etc. For every $D \in V_{i}^{1}$ and every $p=1, \ldots, M+1$ one can find finite families $U_{i+1}^{D, p}$ and $V_{i+1}^{D, p}$ of standard open neighbourhoods of points of $\partial X$ such that:

(a) $\bar{D} \cap \partial X \subset \bigcup V_{i+1}^{D, p}$,

(b) $\left(\forall C \in U_{i+1}^{D, p}\right)\left(\forall A \in U_{i}^{1}\right)$ if $\bar{D} \subset A$ then $\bar{C} \subset A$,

(c) $\left(\forall A \in U_{i+1}^{D, 1}\right) \bar{A} \subset U \backslash B_{N_{i}}$,

(d) $\left(\forall B \in V_{i+1}^{D, p}\right)\left(\exists A \in U_{i+1}^{D, p}\right) \bar{B} \subset A$,

(e) $(\forall p \geq 2)\left(\forall A \in U_{i+1}^{D, p}\right)\left(\exists C \in V_{i+1}^{D, p-1}\right) \overline{\bigcup \operatorname{St}\left(A, U_{i+1}^{D, p}\right)} \subset C$.

Define finite families $V_{r+1}^{p}$ and $U_{r+1}^{p}$ by $V_{r+1}^{p}=\bigcup_{D \in V_{r}^{1}} V_{r+1}^{D, p}$ and $U_{r+1}^{p}=$ $\bigcup_{D \in V_{r}^{1}} U_{r+1}^{D, p}$. Find a natural $N_{i+1}^{\prime}>N_{i}$ such that for every $D \in V_{i}^{1}$ we have $S_{N_{i+1}^{\prime}} \cap \bar{D} \subset \bigcup V_{i+1}^{D, M+1}$. Given an $(M+1)$-simplex $\tau$ of $\mathcal{T}_{i}$, by (iii) we find $D_{\tau} \in V_{i}^{1}$ with $\bar{D}_{\tau} \subset s_{i}(\tau), g_{i}(\partial \tau) \subset D_{\tau}$ and $g_{i}(\tau) \subset \bar{D}_{\tau}$. Observe that then $h_{i}(\partial \tau) \subset D_{\tau} \cap \partial X$ and that, by (b), $\bar{B} \subset s_{i}(\tau)$ for every $B \in V_{i+1}^{D_{\tau}, p}$, $p=1, \ldots, M+1$. Using Lemma 3.3 one can find a map $g_{i+1}^{\prime \tau}: \tau \rightarrow S_{N_{i+1}^{\prime}} \cap \bar{D}_{\tau}$ extending $\left.p_{N_{i+1}^{\prime}} \circ h_{i}\right|_{\partial \tau}: \partial \tau \rightarrow S_{N_{i+1}^{\prime}} \cap D_{\tau}$. By continuity of (every) $g_{i+1}^{\prime \tau}$, one can choose a subdivision $\mathcal{T}_{i+1}$ of the triangulation $\mathcal{T}_{i}$ of $B^{M+1}$ with simplices of diameter at most $2^{-i-1}$, so fine that for every 1-simplex $\sigma$ of $\mathcal{T}_{i+1}$ contained in suitable $\tau$ there exists $B \in V_{i+1}^{D_{\tau}, M+1}$ such that $g_{i+1}^{\prime \tau}(\partial \sigma) \subset B$. For every vertex $v$ of $\mathcal{T}_{i+1}$ not belonging to $\left|\mathcal{T}_{i}^{(M)}\right|$ one can choose a point $\widetilde{v} \in \partial X$ such that $p_{N_{i+1}^{\prime}}(\widetilde{v})=g_{i+1}^{\prime}(v)$, where $g_{i+1}^{\prime}$ is the union of the maps $g_{i+1}^{\prime \tau}$ over all maximal simplices $\tau$ of $\mathcal{T}_{i}$. For a vertex $v \in\left|\mathcal{T}_{i}^{(M)}\right|$ we put $\widetilde{v}=h_{i}(v)$. Again, by induction assumptions, for any two vertices $v, w$ of $\mathcal{T}_{i+1}$ joined by an edge $\langle v, w\rangle$ contained in $\tau$ and not in $\left|\mathcal{T}_{i}^{(M)}\right|$, and for the corresponding points $\widetilde{v}, \widetilde{w} \in \partial X$, one can find $A \in U_{i+1}^{D_{\tau}, M+1}$ and a map $q:\langle v, w\rangle \rightarrow A \cap \partial X$ such that $q(v)=\widetilde{v}$ and $q(w)=\widetilde{w}$.

Assume we have proved that for any $l$-simplex $\sigma$ of $\mathcal{T}_{i+1}$ contained in $\tau$ and not in $\left|\mathcal{T}_{i}^{(M)}\right|$ there exist $A_{0}, A_{1}, \ldots, A_{l} \in U_{i+1}^{D_{\tau}, M+3-l}$ and maps $q_{0}, q_{1}, \ldots, q_{l}: \partial \sigma \rightarrow\left(A_{0} \cup A_{1} \cup \cdots \cup A_{l}\right) \cap \partial X$ sending $(l-1)$-faces of $\sigma$ into distinct $A_{i}$ 's and coherent on their intersections (we have just checked this for $l=2)$. Since $\partial \sigma \subset \bigcup \operatorname{St}(\kappa)$ for every $(l-1)$-simplex $\kappa$ of $\partial \sigma$, we have $\bigcup_{i=0}^{l} A_{i} \subset \bigcup \operatorname{St}\left(A_{0}\right)$. Thus there exists $B \in V_{i+1}^{D_{\tau}, M+2-l}$ such that $\left(\bigcup_{i=0}^{l} q_{i}\right)(\partial \sigma) \subset B$. Hence, if $l \leq M$, by induction assumptions there exists $A \in U_{i+1}^{D_{\tau}, M+2-l}$ and a map $q: \sigma \rightarrow A \cap \partial X$ extending $\bigcup_{i=0}^{l} q_{i}$. If $l=M+1$, we conclude that for every $(M+1)$-simplex $\sigma$ of $\mathcal{T}_{i+1}$ contained in $\tau$ there exists $B \in V_{i+1}^{D_{\tau}, 1}$ and a map $q: \partial \sigma \rightarrow B \cap \partial X$ such that $q(v)=\widetilde{v}$ for every vertex $v$ of $\sigma$ and $q$ coincides with $h_{i}$ on $\partial \tau \cap \partial \sigma$. By (d), there exists $A \in U_{i+1}^{D_{\tau}, 1}$ 
such that $\bar{B} \subset A$. Define $s_{i+1}: \mathcal{T}_{i+1}^{(M+1)} \rightarrow U_{i+1}^{1}$ setting $s_{i+1}(\sigma)=A$. Observe that since $\bar{D}_{\tau} \subset s_{i}(\tau)$, by (b) we have $\bar{A} \subset s_{i}(\tau)$. In other words, for every $(M+1)$-simplex $\tau$ of $\mathcal{T}_{i}$ and an $(M+1)$-simplex $\sigma \subset \tau$ of $\mathcal{T}_{i+1}$ we have $\overline{s_{i+1}(\sigma)} \subset s_{i}(\tau)$.

Because maps of the form $q$ by definition coincide on intersections of their domains, their union is a well-defined continuous map $h_{i+1}^{\tau}:\left|\mathcal{T}_{i+1}^{(M)}\right| \cap \tau \rightarrow$ $\partial X$. Note that $\left.h_{i+1}^{\tau}\right|_{\partial \tau}=\left.h_{i}\right|_{\partial \tau}$, and that for every $(M+1)$-simplex $\sigma \subset \tau$ of $\mathcal{T}_{i+1}$ there exists $B \in V_{i+1}^{D_{\tau}, 1}$ satisfying $h_{i+1}^{\tau}(\partial \sigma) \subset B \cap \partial X$ and $\bar{B} \subset s_{i+1}(\sigma)$.

Because maps of the form $h_{i+1}^{\tau}$ for different choices of $\tau$ coincide on intersections of their domains, we can define $h_{i+1}:\left|\mathcal{T}_{i+1}^{(M)}\right| \rightarrow U \cap \partial X$ as the union of all those maps. One can find a natural $N_{i+1}>N_{i+1}^{\prime}$ such that for every $\tau$ and every $(M+1)$-simplex $\sigma$ of $\mathcal{T}_{i+1}$ contained in $\tau$ there exists $B \in V_{i+1}^{D_{\tau}, 1}$ with $p_{N_{i+1}} \circ h_{i+1}(\partial \sigma) \subset B \cap S_{N_{i+1}}$ and $\bar{B} \subset s_{i+1}(\sigma)$. By Lemma 3.3, for every such $\sigma$ and $B$ there exists a map $g_{i+1}^{\sigma}: \sigma \rightarrow S_{N_{i+1}} \cap \bar{B}$ extending $\left.p_{N_{i+1}} \circ h_{i+1}\right|_{\partial \sigma}: \partial \sigma \rightarrow S_{N_{i+1}} \cap B$. The union of such maps over all maximal simplices defines a map $g_{i+1}^{\tau}: \tau \rightarrow S_{N_{i+1}} \cap C_{\tau}$, which extends $p_{N_{i+1}} \circ h_{i+1}: \partial \tau \rightarrow S_{N_{i+1}} \cap U$. We define $g_{i+1}: B^{M+1} \rightarrow S_{N_{i+1}} \cap U$ as the union of the maps $g_{i+1}^{\tau}$ over all maximal simplices $\tau$ of $\mathcal{T}_{i}$. Observe that by construction $h_{i+1}, g_{i+1}, \mathcal{T}_{i+1}, V_{i+1}^{1}, U_{i+1}^{1}$ satisfy induction assumptions (i)(iv) so that one can proceed with the following steps of the construction.

To start the construction of $g_{i}$ 's one has to define: $N_{0}, g_{0}, V_{0}^{1}$ and $U_{0}^{1}, \mathcal{T}_{0}$, $h_{0}$ and $s_{0}$. Let $N_{0}$ be a natural number such that $S_{N_{0}} \cap V \supset p_{N_{0}} \circ f\left(S^{M}\right)$. By Lemma 3.3 one can find a map $g_{0}: B^{M} \rightarrow S_{N_{0}} \cap \bar{V}$ extending the map $p_{N_{0}} \circ f: S^{M} \rightarrow S_{N_{0}}$. Then set $V_{0}^{1}=\{V\}, U_{0}^{1}=\{U\}, \mathcal{T}_{0}$ the triangulation of $B^{M+1}$ consisting of one $(M+1)$-simplex, $h_{0}=f$ and $s_{0}(\sigma)=U$ for every $\sigma \in \mathcal{T}_{0}^{(M)}$. It is obvious that conditions (i)-(iv) are satisfied.

The rest of the proof mimics the proof of Lemma 3.4 with necessary changes (replace $\mathcal{T}_{k}^{(1)}$ by $\mathcal{T}_{k}^{(M+1)}, I$ by $B^{M+1},\{0,1\}$ by $S^{M}$, and $U_{k}$ by $U_{k}^{1}$, for all $k$ ). We limit ourselves to listing the analogues of the claims of the preceding proof.

ClAim 0. $g_{i}(\tau) \subset s_{L}(\tau)$ for $\tau \in \mathcal{T}_{L}^{(M+1)}$ and $i \geq L$.

Claim 1. For every $y \in B^{M+1}$ the limit $\lim _{i \rightarrow \infty} g_{i}(y)$ exists.

Claim 2. $\lim _{i \rightarrow \infty} g_{i}(y) \in U \cap \partial X$.

Claim 3. The formula $g(x)=\lim _{i \rightarrow \infty} g_{i}(x)$ defines a continuous map $g: B^{M+1} \rightarrow U \cap \partial X$. 
Claim 4. The map $g: B^{M+1} \rightarrow U \cap \partial X$ extends $f$.

This completes the proof of Lemma 3.5.

Proposition 3.6. $\partial X$ is $(n-2)$-connected and locally $(n-2)$-connected.

Proof. For the local statement let $x \in \partial X$ and let $W \ni x$ be its open (in $X \cup \partial X$ ) neighbourhood. By Lemma 2.1 one can find standard neighbourhoods $U$ and $V$ of $x$ contained in $W$ and such that $\bar{V} \subset U$. Then by Lemma 3.5 for every $k \in\{0,1, \ldots, n-2\}$ every map $f: S^{k}=\partial B^{k+1} \rightarrow V$ has an extension $g: B^{k+1} \rightarrow U \subset W$. For the global case apply Lemma 3.5 setting $V=U=X \cup \partial X$.

\section{RIGHT-ANGLED BUILDINGS}

Throughout this section $(W, S)$ is a finitely generated right-angled Coxeter system, not necessarily hyperbolic. In Subsection 4.A we assume it to be finite, i.e. $W \simeq(\mathbb{Z} / 2)^{n}, S=\{(1,0, \ldots, 0), \ldots,(0, \ldots, 0,1)\}$.

4.A. Finite right-angled buildings. We will analyse the structure of finite $W$-buildings, as well as maps between such buildings. This will be needed later for the constructions of infinite right-angled buildings and of maps between them. A typical step of those constructions consists of extending a map defined on a subset of a finite residue to the whole residue.

We will treat a building combinatorially, as a set (of chambers) equipped with a family $\left(\sim_{s}\right)_{s \in S}$ of equivalence relations (the adjacency relations). The standard example of a finite $W$-building is a product building: the set of chambers $Y$ is a product $\prod_{s \in S} Y_{s}$, where each $Y_{s}$ is a finite set of cardinality at least 2 (at least 3 if one wants a thick building). Two chambers $\left(y_{s}\right),\left(y_{s}^{\prime}\right)$ are $t$-adjacent if $y_{s}=y_{s}^{\prime}$ for all $s \neq t$. Apartments are of the form $A=$ $\prod_{s \in S} A_{s}$, where each $A_{s}$ is a two-element subset of $Y_{s}$.

It is easy to see that any $(\mathbb{Z} / 2)^{2}$-building $X$ is a product building (we will frequently apply this fact to residues in larger buildings). Indeed, let $S=\{s, t\}$ and let $Y_{s}=X / \sim_{s}, Y_{t}=X / \sim_{t}$. Since any two chambers $x, x^{\prime} \in X$ are contained in some apartment, $[x]_{\sim_{s}}$ and $\left[x^{\prime}\right]_{\sim_{t}}$ always have a common chamber. Therefore, the map $X \ni x \mapsto\left([x]_{\sim_{s}},[x]_{\sim_{t}}\right) \in Y_{s} \times Y_{t}$ is onto. As no two chambers can be simultaneously $s$ - and $t$-adjacent, this map is also injective.

By a morphism between two $W$-buildings (or subsets of such buildings) we mean a map of the sets of chambers preserving the relations. A subset $E$ of a $W$-building $X$ is called star-like (with respect to a chamber $B \in X$ ) if for every $x \in E$ every minimal gallery from $B$ to $x$ is contained in $E$. Notice that $E=\emptyset$ is star-like. 
LEMMA 4.1. Let $X$ be any finite $W$-building, and let $Y$ be a product $W$ building described above. Let $E \subseteq X$ be star-like with respect to a chamber $B$, and let $\psi: E \rightarrow Y$ be a morphism. Then $\psi$ extends to a morphism $\phi: X \rightarrow Y$. Moreover:

(i) If two such extensions coincide on each class $[B]_{\sim_{s}}$, then they are equal.

(ii) If $\phi$ is injective on each class $[B]_{\sim_{s}}$, then $\phi$ is a monomorphism.

(iii) If $\phi$ maps each class $[B]_{\sim_{s}}$ onto $[\phi(B)]_{\sim_{s}}$, then $\phi$ is an epimorphism.

Proof. Let $\pi: X \rightarrow W$ be the $B$-based folding map. Put $X_{k}=$ $\pi^{-1}(\{w \in W \mid \ell(w) \leq k\})$; in particular, $X_{0}=\{B\}$. Let $C=\psi(B)$ if $B \in E$, or let $C$ be an arbitrary chamber of $Y$ if $E=\emptyset$. Put $\phi(B)=C$. Define $\phi$ on $[B]_{\sim_{s}} \backslash(E \cup\{B\})$ to be an arbitrary map to $[C]_{\sim_{s}}$; define $\phi$ on $[B]_{\sim_{s}} \cap E$ to coincide with the restriction of $\psi$; do this for each $s$. Thus we have defined $\phi$ on $\bigcup_{s \in S}[B]_{\sim_{s}}=X_{1}$ so that it coincides with $\psi$ on $X_{1} \cap E$. Inductively on $k$ we will extend $\phi$ to $X_{k}$, and check that the extension coincides with $\psi$ on $X_{k} \cap E$. Suppose this has been done for $X_{k-1}$, for some $k-1 \geq 1$. Let $x \in X, \pi(x)=w, \ell(w)=k$. For $t \in \operatorname{In}(w)$ we denote by $x^{t}$ the chamber in the $t$-residue of $x$ which is closest to $B$. Since $x \sim_{t} x^{t}, \phi(x)$ has to be $t$-adjacent to $\phi\left(x^{t}\right)$ for every $t \in \operatorname{In}(w)$.

Let $\phi\left(x^{t}\right)=\left(y_{s}^{t}\right)_{s \in S}$. Let $t, t^{\prime} \in \operatorname{In}(w)$ be distinct, and let $s \in S, s \neq t, t^{\prime}$. We claim that $y_{s}^{t}=y_{s}^{t^{\prime}}$. Indeed, let $x^{t, t^{\prime}}$ be the chamber in the $\left\{t, t^{\prime}\right\}$-residue of $x$ which is closest to $B$. Since $W$ is right-angled, we have $x^{t, t^{\prime}}=\left(x^{t}\right)^{t^{\prime}}=$ $\left(x^{t^{\prime}}\right)^{t}$; consequently, $x^{t, t^{\prime}} \sim_{t} x^{t^{\prime}}, x^{t, t^{\prime}} \sim_{t^{\prime}} x^{t}$. Therefore $y_{s}^{t}=\phi\left(x^{t, t^{\prime}}\right)_{s}=y_{s}^{t^{\prime}}$. Denote by $y_{s}$ the common value of $y_{s}^{t}, t \neq s$. Clearly, $y=\left(y_{s}\right)_{s \in S}$ is the unique chamber in $Y$ which is $t$-adjacent to $\phi\left(x^{t}\right)$ for each $t \in \operatorname{In}(w)$. Therefore, we have to put $\phi(x)=y$. Notice that if $x \in E$, then $x^{t} \in E$ for all $t \in \operatorname{In}(w)$. Therefore $\psi(x)$ is $t$-adjacent to $\psi\left(x^{t}\right)=\phi\left(x^{t}\right)$ for all $t \in \operatorname{In}(w)$, hence $\psi(x)=$ $y=\phi(x)$. We apply the above procedure to every $x \in X_{k} \backslash X_{k-1}$, and get the required extension.

Now, (i) follows from the construction: after defining $\phi$ on $X_{1}$ we made no choices.

(ii) Let $\pi_{C}: Y \rightarrow W$ be the $C$-based folding map. This map is given by $\pi_{C}(y)=\Pi_{\left\{s \in S \mid C_{s} \neq y_{s}\right\}} s$. We first show that $\phi$ is $\pi-\pi_{C}$-equivariant. Again, this is done by induction on $k$. We have $\pi_{C}(\phi(B))=\pi_{C}(C)=1=\pi(B)$. Then $\phi\left([B]_{\sim_{s}} \backslash\{B\}\right) \subseteq[C]_{\sim_{s}} \backslash\{C\}, \pi\left([B]_{\sim_{s}} \backslash\{B\}\right)=\{s\}=\pi_{C}\left([C]_{\sim_{s}} \backslash\{C\}\right)$, which checks $\pi$-equivariance on $X_{1}$. Let now $x \in X, y=\phi(x), w=\pi(x)$, $\ell(w)=k \geq 2$. For $t \in \operatorname{In}(w)$ we have $\pi\left(x^{t}\right)=w t$, and, by the inductive assumption, $\pi_{C}\left(\phi\left(x^{t}\right)\right)=w t$. It follows that $\left\{s \in S \mid y_{s}^{t} \neq C_{s}\right\}=\operatorname{In}(w) \backslash\{t\}$. Hence $\left\{s \in S \mid y_{s} \neq C_{s}\right\}=\operatorname{In}(w)$ and $\pi_{C}(\phi(x))=w$.

Consequently, if $\phi(x)=\phi(z)$, then $\pi(x)=\pi(z)$. Let $x, z$ be such a pair with the shortest possible $w=\pi(x)$, and let $t \in \operatorname{In}(w)$. Then $\phi\left(x^{t}\right)=\phi\left(z^{t}\right)$, 
since both are the chamber in the $t$-residue of $\phi(x)=\phi(z)$ which is closest to $C$. Since our counterexample to injectivity has shortest $w$, we deduce $x^{t}=z^{t}$ for all $t \in \operatorname{In}(w)$. Notice that $\ell(w) \geq 2(\pi$-equivariance and injectivity on all $[B]_{\sim_{s}}$ imply that $\phi$ is injective on $\left.X_{1}\right)$. Let $t, t^{\prime}$ be two distinct elements of $\operatorname{In}(w)$. Then $x \sim_{t} x^{t}=z^{t} \sim_{t} z$ and $x \sim_{t^{\prime}} x^{t^{\prime}}=z^{t^{\prime}} \sim_{t^{\prime}} z$, so that $x$ is both $t$ - and $t^{\prime}$-adjacent to $z$. This is possible in a building only if $x=z$.

(iii) By induction on $k$ we will prove that for any $(x, u) \in X_{k} \times S$ the $\operatorname{map} \phi:[x]_{\sim_{u}} \rightarrow[\phi(x)]_{\sim_{u}}$ is surjective. The statement is true for $k=0$ by assumption. Let $x \in X_{k}, \pi(x)=w, \ell(w)=k$, and let $u \in S$. We can assume that $\ell(w u)=k+1$-otherwise $x^{u} \in X_{k-1},[x]_{\sim_{u}}=\left[x^{u}\right]_{\sim_{u}}$ and the statement for $(x, u)$ is true by the inductive assumption applied to $\left(x^{u}, u\right)$. Pick a $t \in \operatorname{In}(w)$. Let $y=\left(y_{s}\right) \in[\phi(x)]_{\sim_{u}}$. We then have $\phi(x)_{s}=y_{s}$ for $s \neq u$, and $\phi\left(x^{t}\right)_{s}=y_{s}$ for $s \neq u, t$. Let $z_{s}=y_{s}$ for $s \neq t, z_{t}=\phi\left(x^{t}\right)_{t}$. Then $z=\left(z_{s}\right) \sim_{t} \phi\left(x^{t}\right)$, hence (by the inductive assumption for $\left(x^{t}, u\right)$ ) there exists $x^{\prime} \in\left[x^{t}\right]_{\sim_{u}}$ such that $\phi\left(x^{\prime}\right)=z$. Observe that in the $\{u, t\}$-residue of $x^{t}$ there is a unique element $x^{\prime \prime}$ which is $u$-adjacent to $x$ and $t$-adjacent to $x^{\prime}$, while $y$ is the unique chamber in $Y$ which is $u$-adjacent to $\phi(x)$ and $t$-adjacent to $z$. Hence, $\phi\left(x^{\prime \prime}\right)=y$, where $x^{\prime \prime} \in[x]_{\sim_{u}}$.

It follows that the image of $\phi$ is closed under all adjacency relations, hence it is equal to $Y$.

\section{Corollaries}

1. One can take $E=\bigcup_{s \in S}[B]_{\sim_{s}}$, choose an arbitrary chamber $\psi(B) \in Y$ and for each $x \in[B]_{\sim_{s}}$ pick an arbitrary $\psi(x) \in[\psi(B)]_{\sim_{s}}$; every such $\psi$ extends to a unique morphism.

2. Let $E=\bigcup_{s \in S}[B]_{\sim_{s}}$, let $Y_{s}=[B]_{\sim_{s}}$. Put $\psi(B)=(B)_{s \in S}$. For $x \sim_{s} B$ put $\psi(x)_{t}=B$ for $t \neq s, \psi(x)_{s}=x$. Then the extension $\phi: X \rightarrow Y$ is an isomorphism. Thus, any finite $W$-building is isomorphic to a product building. Therefore, Lemma 4.1 holds with $Y$ replaced by any finite $W$-building.

3. A corollary of the proof: every monomorphism of finite $W$-buildings $\phi: X \rightarrow Y$ is $\pi$-equivariant (where $\pi: X \rightarrow W$ is a folding map based at an arbitrary chamber $x \in X$, and $\pi: Y \rightarrow W$ the $\phi(x)$-based folding map).

\section{B. Maps of infinite right-angled buildings}

Definition. A standard $W$-building is a set $X$ (of chambers) equipped with: (a) a family $\left(\sim_{s}\right)_{s \in S}$ of equivalence relations with finite equivalence classes; (b) a morphism $\pi: X \rightarrow W$, called the folding map, such that the following are satisfied: 
(i) $(\forall x \in X)(\forall s \in S)\left(\exists x^{\prime} \in X\right)\left(x \sim_{s} x^{\prime} \wedge x \neq x^{\prime}\right)$.

(ii) $\pi^{-1}(1)$ has one element (denoted $B$ and called the base chamber).

(iii) Let $x \in X, T=\operatorname{In}(\pi(x)), w=\pi(x)$. Then $\operatorname{Res}(x, T)$ is a finite rightangled building and the map $\operatorname{Res}(x, T) \ni x^{\prime} \mapsto\left(w w_{T}\right)^{-1} \pi\left(x^{\prime}\right) \in W_{T}$ is a folding map of that building (where $w_{T}$ is the longest element in $\left.W_{T}\right)$.

It is pretty clear that any locally finite $W$-building with any folding map is a standard $W$-building. In particular, condition (iii) follows from property (F3) stated in Section 1. More specifically, $\operatorname{Res}(x, T)$ is mapped by $\pi$ onto the coset $w W_{T}$ of $W_{T} ; w=\pi(x)$ is the longest element of $w W_{T}$, therefore $w w_{T}$ is the shortest element of $w W_{T}$. Let $y$ be the shortest chamber in $\operatorname{Res}(x, T)$ (as in (F3)); then $\pi(y)=w w_{T}$. The $y$-based folding map of $\operatorname{Res}(x, T)$ is the composition of (restricted) $\pi$ and the left multiplication in $W$ that moves the coset $w W_{T}$ to $W_{T}$ and the element $\pi(y)$ to 1 . This left multiplication is the left multiplication by $\pi(y)^{-1}=\left(w w_{T}\right)^{-1}$.

\section{REMARKS}

1. Later we will prove that a standard building is in fact a building.

2. The residue $\operatorname{Res}(x, T)$ in condition (iii) intersects $\pi^{-1}\left(w w_{T}\right)$ in one chamber, to be called the shortest chamber of $\operatorname{Res}(x, T)$. The folding map in condition (iii) is based at that chamber.

3. Conditions (i) and (iii) together imply that for every $x \in X$ and every spherical $T \subseteq S$ the residue $\operatorname{Res}(x, T)$ is a finite $W_{T}$-building, and the restriction of $\pi$ composed with left multiplication by the inverse of the shortest element of $\pi(\operatorname{Res}(x, T))$ is a folding map of that building.

4. It follows from the previous remark that if $t \in \operatorname{In}(\pi(x))$ then the intersection $\pi^{-1}(\pi(x) t) \cap[x]_{\sim_{t}}$ consists of a unique element (to be denoted $\left.x^{t}\right)$.

Definition. A local $W$-building is a set $Y$ (of chambers) equipped with a family $\left(\sim_{s}\right)_{s \in S}$ of equivalence relations, such that:

(a) for every $y \in Y$ and every spherical $T \subseteq S, \operatorname{Res}(y, T)$ is a finite $W_{T^{-} \text {building; }}$

(b) $Y$ is gallery connected, i.e., for every $y, y^{\prime} \in Y$ there exists a gallery from $y$ to $y^{\prime}$ : a sequence $y_{0}=y, y_{1}, \ldots, y_{k}, y_{k+1}=y^{\prime}$ such that $y_{i} \sim_{s_{i}}$ $y_{i+1}$ for some $s_{i} \in S$, where $i=0,1, \ldots, k$.

REmark. A standard $W$-building is a local $W$-building. Condition (a) follows from the third remark above. To check condition (b), we show that there exists a gallery from an arbitrary chamber $x$ to $B$. Let $\pi(x)=w$. It follows from the fourth remark above that a minimal gallery in $W$ from $w$ to 1 can be lifted to a gallery in $X$ from $x$ to some chamber in $\pi^{-1}(1)$; however, $\pi^{-1}(1)=\{B\}$. 
The following theorem is rather weak. The proof will give us an idea of what should really be done.

THEOREM 4.2. Let $X$ be a standard $W$-building, and let $Y$ be a local $W$-building. Then there exists a morphism $\phi: X \rightarrow Y$.

Proof. Choose a well-ordering $<$ on $X$ such that each initial segment $X_{<x}$ is star-like (with respect to $B$ ). We define $\phi$ inductively. To start, we pick any $y \in Y$ and declare $\phi(B)=y$. Suppose $\phi: X_{<x} \rightarrow Y$ has already been defined. Let $T=\operatorname{In}(\pi(x))$. Since $X_{<x}$ is star-like, so is $X_{<x} \cap \operatorname{Res}(x, T)$ (in $\operatorname{Res}(x, T)$, with respect to the shortest element $x_{0}$ of that residue). Since $\phi$ is a morphism, it maps $X_{<x} \cap \operatorname{Res}\left(x_{0}, T\right)$ into $\operatorname{Res}\left(\phi\left(x_{0}\right), T\right)$; this restriction can, by Lemma 4.1 , be extended to $\eta: \operatorname{Res}\left(x_{0}, T\right) \rightarrow \operatorname{Res}\left(\phi\left(x_{0}\right), T\right)$ (since $Y$ is a local building, $\operatorname{Res}\left(\phi\left(x_{0}\right), T\right)$ is a finite $W_{T}$-building; therefore, Lemma 4.1 can indeed be applied). We put $\phi(x)=\eta(x)$. Since all chambers in $X_{<x}$ which are adjacent to $x$ belong to $\operatorname{Res}\left(x_{0}, T\right)$ (where $\phi$ coincides with $\eta$ ), the extended $\phi: X_{<x} \cup\{x\} \rightarrow Y$ is a morphism.

Notice that, in the construction of $\phi$ described in the proof, if $\operatorname{In}(\pi(x))$ has at least two elements, then $\phi(x)$ is uniquely determined by $\left.\phi\right|_{X_{<x}}$. In fact, if $u, t \in \operatorname{In}(\pi(x))$, then $\phi(x)$ is uniquely determined by $\phi\left(x^{t}\right)$ and $\phi\left(x^{u}\right)$ : it is the unique chamber $u$-adjacent to $\phi\left(x^{t}\right)$ and $t$-adjacent to $\phi\left(x^{u}\right)$ (as in the proof of Lemma 4.1). If, on the other hand, $\operatorname{In}(\pi(x))=\{s\}$, then $\phi(x)$ can be freely chosen in $\left[\phi\left(x_{0}\right)\right]_{\sim_{s}}$. These observations are basic for the next theorem.

Let $X$ be a standard $W$-building, and let $Y$ be a local $W$-building. We say that a morphism $\phi: X \rightarrow Y$ is a local monomorphism (resp. local epimorphism, covering map) if for every $x \in X$ and every spherical $T \subseteq S$ the residue $\operatorname{Res}(x, T)$ is injectively (resp. surjectively, bijectively) mapped by $\phi$ to $\operatorname{Res}(\phi(x), T)$.

Definition. The root set of a standard $W$-building $X$ is

$$
R(X)=\{(x, s) \in X \times S \mid \operatorname{In}(\pi(x) s)=\{s\}\} .
$$

THEOREM 4.3. Let $X$ be a standard $W$-building, let $Y$ be a local $W$ building, and let $\phi: X \rightarrow Y$ be a morphism. Let $R=R(X)$ be the root set of $X$.

(i) The map $\phi$ is uniquely determined by $\phi(B)$ and the restrictions of $\phi$ to $[x]_{\sim_{s}}$, over all $(x, s) \in R$.

(ii) If all the above restrictions are injective, then $\phi$ is a local monomorphism.

(iii) If for each $(x, s) \in R, \phi$ maps $[x]_{\sim_{s}}$ onto $[\phi(x)]_{\sim_{s}}$, then $\phi$ is a local epimorphism and a surjection. 
(iv) If , for each $(x, s) \in R, \phi$ maps $[x]_{\sim_{s}}$ bijectively onto $[\phi(x)]_{\sim_{s}}$, then $\phi$ is a covering map.

Proof. (i) Let $\phi_{1}, \phi_{2}: X \rightarrow Y$ coincide on $B$ and on each $[x]_{\sim_{s}},(x, s) \in R$. Suppose that $x \in X$ is a chamber with shortest $w=\pi(x)$ such that $\phi_{1}(x)$ $\neq \phi_{2}(x)$. If $\operatorname{In}(w)=\emptyset$, then $w=1$ and $x=B$, a contradiction. If $\operatorname{In}(w)$ $=\{s\}$, then $\left(x^{s}, s\right) \in R, x \in\left[x^{s}\right]_{\sim_{s}}$, a contradiction again. If $T=\operatorname{In}(w)$ has at least two elements, then, by Lemma 4.1(i) applied to $\operatorname{Res}(x, T), \phi_{1}(x)$ and $\phi_{2}(x)$ are uniquely determined by $\left.\phi_{1}\right|_{X_{k}}=\left.\phi_{2}\right|_{X_{k}}$ (where $\left.k=\ell(w)-1\right)$, so that they coincide.

(ii) Suppose not. Let $x_{0} \in X$ be an element of $X$ with the shortest possible $w=\pi\left(x_{0}\right)$, such that for some spherical $T$ the restriction of $\phi$ to $\operatorname{Res}\left(x_{0}, T\right)$ is not injective. By Lemma 4.1(ii), there exists $t \in T$ and chambers $x, x^{\prime} \sim_{t} x_{0}$ such that $\phi(x)=\phi\left(x^{\prime}\right)$. If $\operatorname{In}(w t) \neq\{t\}$, then $\operatorname{Res}(x, \operatorname{In}(w t))$ is a residue on which $\phi$ is injective, and whose shortest chamber is shorter than $x_{0}$, a contradiction. In the case $\operatorname{In}(w t)=\{t\}$ we have $\left(x_{0}, t\right) \in R$ and $x, x^{\prime} \in\left[x_{0}\right]_{\sim_{t}}$, so that $\phi(x) \neq \phi\left(x^{\prime}\right)$, a contradiction.

(iii) Suppose that $\phi$ is not a local epimorphism. Let $\operatorname{Res}\left(x_{0}, T\right)$ be a counterexample with shortest $w=\pi\left(x_{0}\right)$. Then, by Lemma 4.1(iii), there is a $t \in T$ such that $\phi:\left[x_{0}\right]_{\sim_{t}} \rightarrow\left[\phi\left(x_{0}\right)\right]_{\sim_{t}}$ is not onto. As in the proof of (ii) we see that $\operatorname{In}(w t)=\{t\}$. Therefore $\left(x_{0}, t\right) \in R$, a contradiction.

Since the image of a local epimorphism is closed under the adjacency relations, and since $Y$ is gallery connected, we have $\phi(X)=Y$.

Finally, (iv) follows from (ii) and (iii).

4.C. Construction. In this subsection we present a construction of a general standard $W$-building. Let $W=\left\{w_{1}=1, w_{2}, \ldots\right\}$ be a numbering of elements of $W$ such that each $W_{k}=\left\{w_{1}, \ldots, w_{k}\right\}$ is a star-like subset of $W$ (with respect to 1 ). The process of building $X$ is inductive. At the $k$ th step we construct the part $X_{k}$ of $X$ which is going to be the preimage of $W_{k}$ under the folding map. To get $X_{k}$ from $X_{k-1}$ we need to attach the chambers that fold to $w_{k}$. Such a chamber $x$ is contained in a finite residue $\operatorname{Res}\left(x, \operatorname{In}\left(w_{k}\right)\right)$ which is isomorphic to a product building and whose large part is contained in $X_{k-1}$. Thus, $X_{k}$ is obtained from $X_{k-1}$ by gluing to it product buildings that will become $\operatorname{Res}\left(x, \operatorname{In}\left(w_{k}\right)\right)$ for $x \in \pi^{-1}\left(w_{k}\right)$.

We now proceed to the details. We would like to construct, by induction on $k$, sets $X_{k}$ with equivalence relations $\left(\sim_{s}^{k}\right)_{s \in S}$, together with morphisms $\pi_{k}: X_{k} \rightarrow W_{k}$, such that:

(i) $X_{k-1} \subseteq X_{k}$.

(ii) Restricting $\sim_{s}^{k}$ from $X_{k} \times X_{k}$ to $X_{k-1} \times X_{k-1}$ yields $\sim_{s}^{k-1}$. (Therefore, we simply use $\sim_{s}$.)

(iii) $\left.\pi_{k}\right|_{X_{k-1}}=\pi_{k-1}$. (Again, we often denote $\pi_{k}$ simply by $\pi$.) 
(iv) $\pi_{k}^{-1}\left(W_{k-1}\right)=X_{k-1}$.

(v) Let $x \in X_{k}, T=\operatorname{In}(\pi(x)), w=\pi(x)$. Then $\operatorname{Res}_{k}(x, T)$ is a finite right-angled building and the $\operatorname{map}_{\operatorname{Res}_{k}}(x, T) \ni x^{\prime} \mapsto\left(w w_{T}\right)^{-1} \pi\left(x^{\prime}\right)$

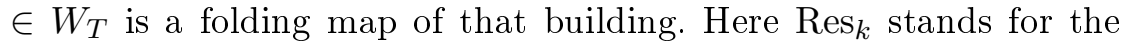
residue in $X_{k}$.

Finally, we will obtain a standard $W$-building $X=\bigcup_{k} X_{k}$ with the folding map $\pi=\bigcup_{k} \pi_{k}$. In fact, at the $k$ th step we will construct not only $X_{k}$ and $\pi_{k}$, but also the following additional data:

(a) an integer $q_{x, s} \geq 1$ for each $(x, s) \in X_{k} \times S$;

(b) for each $u \in W$ such that $u w_{U} \in W_{k}$ (where $U=\operatorname{In}(u)$ ) and each $y \in \pi^{-1}\left(u w_{U}\right)$ : a $\left(u w_{U}\right)^{-1} \pi_{k^{-}} \pi_{y, U^{-}}$equivariant monomorphism $\phi_{y, U}: \operatorname{Res}_{k}(y, U) \rightarrow Y_{y, U}$. Here $Y_{y, U}$ is a product $W_{U}$-building with the $s$-factor $Y_{y, s}$ of cardinality $q_{y, s}+1$, and $\pi_{y, U}: Y_{y, U} \rightarrow W_{U}$ is the $\phi_{y, U}(y)$-based folding map. We will usually briefly say that $\phi_{y, U}$ is $\pi$-equivariant.

The numbers $q_{x, s}$ are subject to extra conditions:

(vi) If $q_{z, s}$ and $q_{z^{\prime}, s}$ are defined and $z \in \operatorname{Res}_{k}\left(z^{\prime}, T\right)$ for a spherical $T \subseteq S$ containing $s$, then $q_{z, s}=q_{z^{\prime}, s}$.

(vii) If $y \in X_{k}$ and $\{\pi(y), \pi(y) s\} \subseteq W_{k}$, then $\operatorname{Res}_{k}(y, s)$ has $q_{y, s}+1$ elements.

In the first step, $X_{1}=\{B\}$, and we choose the numbers $q_{B, s}$ and the maps $\phi_{B, T}:\{B\} \rightarrow Y_{B, T}$ arbitrarily.

Suppose that we have already constructed everything promised for $k-1$. Let $w=w_{k}$, let $T=\operatorname{In}(w)$. The set $X_{k}$ is obtained from $X_{k-1}$ by gluing $Y_{x, T}$, for all $x \in \pi^{-1}\left(w w_{T}\right)$, via the maps $\phi_{x, T}$. Throughout the proof, $x$ will be a generic notation for an element of $\pi^{-1}\left(w w_{T}\right)$.

LEMma. Let $x_{1}, x_{2} \in \pi^{-1}\left(w w_{T}\right), x_{1} \neq x_{2}$. Then

$$
\operatorname{Res}_{k-1}\left(x_{1}, T\right) \cap \operatorname{Res}_{k-1}\left(x_{2}, T\right)=\emptyset .
$$

Proof. Suppose not; then $x_{2} \in \operatorname{Res}_{k-1}\left(x_{1}, T\right)$. The map $\phi_{x_{1}, T}$ being $\pi$ equivariant, we have $\phi_{x_{1}, T}\left(x_{2}\right)=\phi_{x_{1}, T}\left(x_{1}\right)$. However, $\phi_{x_{1}, T}$ is injective; hence $x_{1}=x_{2}$, contradiction.

Now, (i) follows from the fact that the gluing maps are injective.

(ii) We have

Claim. If $y, z \in \operatorname{Res}_{k-1}(x, T)$ and $\phi_{x, T}(y) \sim_{t} \phi_{x, T}(z)$, then $y \sim_{t} z$.

Proof. We can assume that $t \in T$, for otherwise $y=z$. Let $\phi=\phi_{x, T}$, $Y=Y_{x, T}$. There are two cases.

(a) $\{\pi(y), \pi(y) t\} \subseteq W_{k-1}$. In that case, due to (vii), $\operatorname{Res}_{k-1}(y, t)$ has $q_{y, t}+1$ elements; $\operatorname{Res}_{Y}(\phi(y), t)$ has $q_{x, t}+1$ elements. However, (vi) 
implies that $q_{x, t}=q_{y, t}\left(y \in \operatorname{Res}_{k-1}(x, T)\right)$; therefore $\phi$ restricts to a bijection between these residues. Since $\phi$ is an injection, this implies that $z \in \operatorname{Res}_{k-1}(y, t)$.

(b) $\pi(y) t=w$. Then $\operatorname{Res}_{Y}(\phi(y), t)$ consists of $\phi(y)$ and chambers which map to $w_{T}$ under $\pi_{x, T}$. Hence either $\phi(z)=\phi(y)$ (and, $\phi$ being injective, we get $z=y$ ) or $\pi_{x, T}(\phi(z))=w_{T}$. In the latter case $\pi$-equivariance of $\phi$ implies $\pi(z)=w$, which contradicts $z \in X_{k-1}$. Claim

By the Claim, the following definition makes sense: $y \sim_{s}^{k} z$ if either $y, z \in X_{k-1}$ and $y \sim_{s}^{k-1} z$, or $y, z \in Y_{x, T}$ for some $x$ and $y \sim_{s} z$ in $Y_{x, T}$.

Finally, we need to check that $\sim_{s}^{k}$ is an equivalence relation, the only nontrivial condition being transitivity: $\left(a \sim_{s} b \wedge b \sim_{s} c\right) \Rightarrow a \sim_{s} c$. The cases $a, b, c \in X_{k-1}$ and $a, b, c \in Y_{x, T}$ are clear. Thus, we can assume that at least one of $a, b, c$ is in $Y_{x, T} \backslash X_{k-1}$ (for some $x$ ). Then we can assume that $s \in T$ (otherwise $a=b=c$ ). If $b \in Y_{x, T} \backslash X_{k-1}$, then $a, c \in Y_{x, T}$ and $a \sim_{s} c$ follows. If not, we can assume $a \in Y_{x, T} \backslash X_{k-1}, b \in Y_{x, T} \cap X_{k-1}=\operatorname{Res}_{k-1}(x, T)$. Now if $c \in X_{k-1}$, then $c \sim_{s} b$ implies $c \in \operatorname{Res}_{k-1}(x, T) \subseteq Y_{x, T}$, and $a \sim_{s} c$ follows. If $c \notin X_{k-1}$, then $c \in Y_{x^{\prime}, T}$ (for some $x^{\prime} \in \pi^{-1}(w)$ ). It follows that $b \in Y_{x^{\prime}, T} \cap Y_{x, T}$, hence, in view of the Lemma, $x=x^{\prime}$ and $a, b, c \in Y_{x, T}$.

(iii) We define $\pi_{k}$ as follows: if $y \in X_{k-1}$, then $\pi_{k}(y)=\pi_{k-1}(y)$; if $y \in Y_{x, T}$ we put $\pi_{k}(y)=w w_{T} \pi_{x, T}(y)$. This definition is correct because of the $\pi$-equivariance of $\phi_{x, T}$. Condition (iii) is clear.

(iv) Suppose that $y \in Y=Y_{x, T}$, but $y$ is not in the image of $\phi=\phi_{x, T}$. We claim that $\pi(y)=w$, or equivalently, that $\pi_{x, T}(y)=w_{T}$. Suppose not; let $y$ be a counterexample with shortest $u=\pi_{x, T}(y)$. Notice that $u \neq 1$, because $\pi_{x, T}^{-1}(1)=\{\phi(x)\}$. Let $t \in \operatorname{In}(u)$, and let $y^{t}=\phi(z)$. As in (ii), we have $q_{x, t}=q_{z, t}$. Moreover, $\pi(z)=w w_{T} u t$ and $\pi(z) t=w w_{T} u$ belong to $W_{k-1}$, so that $\operatorname{Res}_{k-1}(z, t)$ has cardinality $q_{z, t}+1$, the same as $\operatorname{Res}_{Y}\left(y^{t}, t\right)$. Therefore $\phi$ maps $\operatorname{Res}_{k-1}(z, t)$ bijectively onto $\operatorname{Res}_{Y}\left(y^{t}, t\right)$, and $y$ is in the image of $\phi$, a contradiction.

(v) The new residues to be checked are $\operatorname{Res}_{k}(y, T)$, for $y \in Y_{x, T}, \pi(y)=w$. But in this case $\operatorname{Res}_{k}(y, T)=Y_{x, T}$, and $\left(w w_{T}\right)^{-1} \pi=\pi_{x, T}$.

(vi) Let $y \in Y_{x, T} \backslash X_{k-1}, s \in S$. If $s \in T$, we put $q_{y, s}=q_{y^{s}, s}$. If $s \notin T$, but there exists $t \in T$ such that $\{t, s\}$ is spherical, then we put $q_{y, s}=q_{y^{t}, s}$. This does not lead to contradictions: if $t^{\prime} \in T$ and $\left\{s, t^{\prime}\right\}$ is spherical, then $\left\{s, t, t^{\prime}\right\}$ is also spherical, and $y^{t^{\prime}} \in \operatorname{Res}_{k-1}\left(y^{t},\left\{s, t, t^{\prime}\right\}\right)$, so that $q_{y^{t^{\prime}, s}}=q_{y^{t}, s}$ by (vi). Finally, if $s \notin T$ and no $t \in T$ commutes with $s$, then we choose $q_{y, s}$ arbitrarily. Observe that this last case occurs exactly when $\operatorname{In}(w s)=\{s\}$.

Now suppose that $z, z^{\prime} \in X_{k}, z \in \operatorname{Res}_{k}\left(z^{\prime}, U\right)$ where $U$ is spherical and $s \in U$. We will show that $q_{z, s}=q_{z^{\prime}, s}$. Let $z=z_{1}, z_{2}, \ldots, z_{m}=z^{\prime}$ be a gallery 
in $X_{k}, z_{i} \sim_{u_{i}} z_{i+1}, u_{i} \in U$. Suppose that some two consecutive chambers $z_{i}, z_{i+1}$ do not belong to $X_{k-1}$. Then they are both in $Y_{x, T}$ for some $x$, and $u_{i} \in T$. We insert $z_{i}^{u_{i}}$ between $z_{i}$ and $z_{i+1}$. Repeating the process we ensure that if $z_{i} \notin X_{k-1}$, then $z_{i-1}, z_{i+1} \in X_{k-1}$ (except $i=0, m$ ). Then we replace each triple $z_{i-1}, z_{i}, z_{i+1}$ with $z_{i} \notin X_{k-1}$ by $z_{i-1}, z_{i-1}^{u_{i}}=z_{i+1}^{u_{i-1}}, z_{i+1}$. We obtain a $U$-gallery from $z$ to $z^{\prime}$ whose all but external chambers lie in $X_{k-1}$. We conclude that $q_{z, s}=q_{z_{1, s}}=q_{z_{m-1}, s}=q_{z^{\prime}, s}$.

(vii) The new residues to be checked are $\operatorname{Res}_{k}(y, s)$ for $y$ such that $\pi(y)=$ $w$ or $\pi(y)=w s$ (where $s \in T$ and $y \in Y_{x, T}$ for some $x$ ). In either case, $\operatorname{Res}_{k}(y, s)=\operatorname{Res}_{Y}(y, s)$ has $q_{x, s}+1$ elements. However, $y \in \operatorname{Res}_{k}(x, T)$ so that, by (vi), $q_{x, s}=q_{y, s}$.

Finally, we need to construct or extend some of the maps $\phi_{y, U}$.

Some cases are easy. If $u=w$, then $U=T$ and $y \in \pi^{-1}\left(w w_{T}\right)$. Then $\operatorname{Res}_{k}(y, T)=Y_{y, T}$ and we put $\phi_{y, T}=\operatorname{Id}_{Y_{y, T}}$. If $u w_{U}=w$, then we choose $\phi_{y, U}:\{y\} \rightarrow Y_{y, U}$ arbitrarily. If $w \notin u W_{U}$, then we do not change $\phi_{y, T}$.

Thus, we can assume that $w \in u W_{U}$, but $w \neq u w_{U}$. Then $w=u w_{U} u_{1} \ldots u_{k}$ for some pairwise different $u_{1}, \ldots, u_{k} \in U$. We have $u_{i} \in \operatorname{In}(w)=T$, so that $w \in u w_{U} W_{U \cap T}$, or equivalently, $u w_{U} \in w W_{U \cap T}$. Since $u w_{U}$ is the shortest element in $u W_{U}=w W_{U}$, it is also the shortest element in $w W_{U \cap T}$.

Claim. There is an $x \in \pi^{-1}\left(w w_{T}\right)$ such that $y \in Y_{x, T}$.

Proof. Since $u w_{U} \in w W_{U \cap T} \subseteq w W_{T}=w w_{T} W_{T}$, there is a $T^{\prime} \subseteq T$ such that $u w_{U}=w w_{T} w_{T^{\prime}}$. Moreover, $T^{\prime} \subseteq \operatorname{In}\left(u w_{U}\right)$, therefore $w w_{T} \in$ $u w_{U} W_{T^{\prime}} \subseteq u w_{U} W_{\operatorname{In}\left(u w_{U}\right)}=\pi(y) W_{\operatorname{In}(\pi(y))}$. By $(\mathrm{v})$, there is an $x \in \pi^{-1}\left(w w_{T}\right)$ $\cap \operatorname{Res}_{k-1}\left(y, T^{\prime}\right)$ (the folding map, when restricted to a residue, is onto a suitable coset of the corresponding special subgroup of the Coxeter group). Then $y \in \operatorname{Res}_{k}\left(x, T^{\prime}\right) \subseteq \operatorname{Res}_{k}(x, T)=Y_{x, T}$. Claim $_{\text {Cain }}$

Let $Y_{y, U \cap T}=\operatorname{Res}_{Y_{x, T}}(y, U \cap T)$. Since $\operatorname{Res}_{k-1}(y, U) \cap Y_{y, U \cap T}=Y_{y, U \cap T} \backslash$ $\pi^{-1}(w)$, it is a star-like set in the building $Y_{y, U \cap T}$ (with respect to $y$ ), therefore $\phi_{y, U}$ extends to a monomorphism $\psi: Y_{y, U \cap T} \rightarrow Y_{y, U}$. Gluing $\phi_{y, U}$ with $\psi$ we get an extended map $\phi_{y, U}: \operatorname{Res}_{k-1}(y, U) \cup Y_{y, U \cap T} \rightarrow Y_{y, U}$. We claim that this map is injective: indeed, $\psi$ is $\pi$-equivariant as a monomorphism of buildings, and hence the extended map is $\pi$-equivariant. Furthermore, if $z \in \operatorname{Res}_{k-1}(y, U)$ and $z^{\prime} \in Y_{y, U \cap T} \backslash \operatorname{Res}_{k-1}(y, U)$, then $\pi(z) \neq w=\pi\left(z^{\prime}\right)$, therefore $\phi_{y, U}(z) \neq \phi_{y, U}\left(z^{\prime}\right)$. Now it is enough to observe that $\phi_{y, U}$ is injective on $\operatorname{Res}_{k-1}(y, U)$ and that $\psi$ is injective.

Finally, we claim that $\operatorname{Res}_{k-1}(y, U) \cup Y_{y, U \cap T}=\operatorname{Res}_{k}(y, U)$. Since $\pi$ is a morphism, we know that $\operatorname{Res}_{k}(y, U) \subseteq \pi^{-1}\left(u w_{U} W_{U} \cap W_{k}\right)$. Any $U$-gallery in $X_{k}$ starting at $y$ and ending at $z \notin \pi^{-1}(w)$ can be modified, using the technique from the proof of (vi), to a $U$-gallery not containing chambers from $\pi^{-1}(w)$. This means that $\operatorname{Res}_{k}(y, U) \cap X_{k-1}=\operatorname{Res}_{k-1}(y, U)$. Suppose 
now that $z \in \pi^{-1}(w) \cap \operatorname{Res}_{k}(y, U)$. Then $\operatorname{Res}_{k}(z, U \cap T) \subseteq \operatorname{Res}_{k}(y, U)$, $\operatorname{Res}_{k}(z, U \cap T) \backslash \pi^{-1}(w) \subseteq \operatorname{Res}_{k-1}(y, U)$. In particular, $\operatorname{Res}_{k}(z, U \cap T)$ has a unique shortest element, lying in $\pi^{-1}\left(u w_{U}\right) \cap \operatorname{Res}_{k-1}(y, U)$. But the latter set equals $\{y\}$, because $\phi_{y, U}: \operatorname{Res}_{k-1}(y, U) \rightarrow Y_{y, U}$ is a $\pi$-equivariant monomorphism. Therefore $y \in \operatorname{Res}_{k}(z, U \cap T), z \in Y_{y, U \cap T \text {. }}$

REMARK. Notice that we were free to choose $q_{x, s}$ exactly for $(x, s)$ in the root set of $X$.

\section{D. Uniqueness and lattices}

TheOREM 4.4. For every local $W$-building $Y$ and any chamber $y \in Y$ there exists a standard $W$-building $X$ and a covering map $\phi: X \rightarrow Y$ with $\phi(B)=y$.

Proof. We perform the construction of $X$ as in Subsection 4.C, together with the construction of $\phi$ as in the proof of Theorem 4.2. We put $X_{1}=\{B\}$ and $\phi(B)=y$. Whenever we construct a chamber $x$ such that a choice of $q_{x, s}$ is needed for some $s$, we choose $q_{x, s}=\left|[\phi(x)]_{\sim_{s}}\right|-1$. The pair $(x, s)$ will belong to the root set of $X$. Later, when $\pi^{-1}(\pi(x) s)$ is constructed, we are free to choose $\phi:[x]_{\sim_{s}} \rightarrow[\phi(x)]_{\sim_{s}}$ (extending $x \mapsto \phi(x)$ ); we choose a bijection. By Theorem 4.3(iv) we obtain a covering map.

To talk about universal covers it is convenient to switch to the topological category (and back). The geometric realisation $|X|$ of a local $W$-building $X$ is the geometric realisation of the poset of finite type residues in $X$ (i.e. $T$-residues for all spherical $T)$. One can label each vertex in $|X|$ with the type of the corresponding residue. Let us give another description of $|X|$. Let $L$ be the finite simplicial complex with vertex set $L^{(0)}=S$, a set of generators spanning a simplex in $L$ if and only if they pairwise commute. We denote by $L^{\prime}$ the first barycentric subdivision of $L$, and by $C L^{\prime}$ the cone over $L^{\prime}$. Then $|X|$ is $X \times C L^{\prime} / \sim$, where $(x, p) \sim\left(x^{\prime}, p^{\prime}\right) \Leftrightarrow p=p^{\prime}$ and $x^{\prime} \in \operatorname{Res}(x, S(p))$; here $S(p)=\left\{s \in L^{(0)}\left|\exists \sigma \in L^{\prime}, s \in \sigma, p \in\right| \sigma \mid\right\}$. If $p: \widetilde{|X|} \rightarrow|X|$ is any covering of $X$, then $\widetilde{|X|}$ is in fact a geometric realisation of a local $W$-building $\widetilde{X}: \widetilde{X}$ is the preimage under $p$ of the set of $\emptyset$-labelled vertices of $|X| ; \widetilde{x} \sim_{s} \widetilde{x}^{\prime} \Leftrightarrow$ there exists a vertex $v \in \widetilde{|X|}$, joined by edges to $\widetilde{x}$ and to $\widetilde{x}^{\prime}$, and such that $p(v)$ is of type $\{s\}$. Notice that for any spherical $U \subseteq S$, and any residue $R$ in $X$ of type $U$, the set $\left|X_{\leq R}\right|$ is contractible (as a cone with apex $R$ ), hence its preimage under $p$ is a disjoint union of its homeomorphic copies. Therefore, $\widetilde{X}$ is indeed a local $W$-building. We say that $\widetilde{X}$ is the universal cover of $X$ if $\widetilde{|X|}$ is the universal cover of $|X|$. It is clear that morphisms of local $W$-buildings induce simplicial label-preserving maps, and covering maps of local $W$-buildings induce simplicial covering maps. 
THEOREM 4.5. A standard $W$-building is a building.

Proof. We begin with

LEMMA 4.6. The geometric realisation of a standard $W$-building is contractible.

Proof. The argument follows Serre's proof for buildings [Se]. Let $W=$ $\left\{w_{1}=1, w_{2}, \ldots\right\}$ be a numbering of elements of $W$ such that each set $W_{k}=$ $\left\{w_{1}, \ldots, w_{k}\right\}$ is star-like. Let $X_{k}=\pi^{-1}\left(W_{k}\right)$. The strategy is to show that $\left|X_{k}\right|$ deformation retracts onto $\left|X_{k-1}\right|$. To do this, it is enough to check that each chamber $|x|$ in $\left|X_{k} \backslash X_{k-1}\right|$ deformation retracts onto $|x| \cap\left|X_{k-1}\right|$. Let $T=\operatorname{In}\left(w_{k}\right)$; then the pair $\left(|x|,|x| \cap\left|X_{k-1}\right|\right)$ is isomorphic to $\left(K, K^{T}\right)$, where $K^{T}=\bigcup_{t \in T} K_{t}$. Since $K$ is a cone over $K^{S}$, it is contractible. It is checked in [D1] that $K^{T}$ is contractible for all spherical $T$. It follows that $K$ deformation retracts onto $K^{T}$. Lemma 4.6

Let now $X$ be a standard $W$-building.

Lemma 4.7. Suppose that $\sigma: W \rightarrow X$ is a local monomorphism such that $\sigma(1)=B$. Then $\sigma$ is a section of $\pi$ (i.e., $\pi \circ \sigma=\operatorname{Id}_{W}$ ).

Proof. We argue by contradiction. Let $w \in W$ be the shortest element such that $\pi(\sigma(w)) \neq w$. Notice that $w \neq 1$, because $\pi(\sigma(1))=\pi(B)=1$; consequently, $\operatorname{In}(w) \neq \emptyset$. Let $s \in \operatorname{In}(w)$; then $\pi(\sigma(w s))=w s$. The map $(w s)^{-1} \pi: \operatorname{Res}(\sigma(w s), s) \rightarrow W_{\{s\}}$ is a folding map (cf. Remark 3 after the definition of a standard building), so that $\pi(\sigma(w s))=w s$ while for any $x \in \operatorname{Res}(\sigma(w s), s) \backslash\{\sigma(w s)\}$ we have $\pi(x)=w$. Since $w \in \operatorname{Res}(w s, s)$, we have $\sigma(w) \in \operatorname{Res}(\sigma(w s), s)$; but $\sigma$ is injective on $\operatorname{Res}(w s, s)$ so that $\sigma(w) \neq \sigma(w s)$. Therefore $\pi(\sigma(w))=w$, a contradiction. -Lemma 4.7

We define an apartment in $X$ as the image of any monomorphism $\sigma$ : $W \rightarrow X$. Notice that if $B \in \sigma(W)$, then we can modify $\sigma$ by precomposing it with left multiplication by $\sigma^{-1}(B)$, so as to have $\sigma(1)=B$. Therefore, every apartment containing $B$ is the image of a section of $\pi$.

Let us make two observations that will be used for checking (B2) and (B3). First, observe that if $\sigma, \sigma^{\prime}$ are sections of $\pi$, and $\sigma(W), \sigma^{\prime}(W)$ are two apartments containing $B$ and a chamber $x$, then $\sigma^{\prime} \circ \pi: \sigma(W) \rightarrow \sigma^{\prime}(W)$ is an isomorphism fixing $B$ and $x$. Next, if $\sigma, \sigma^{\prime}$ are sections of $\pi$, and $\sigma(W)$, $\sigma^{\prime}(W)$ are apartments containing $B$ and intersecting an $s$-residue $R$, then $\sigma^{\prime} \circ \pi: \sigma(W) \rightarrow \sigma^{\prime}(W)$ is an isomorphism fixing $B$ and mapping $R \cap \sigma(W)$ to $R \cap \sigma^{\prime}(W)$. Indeed, $\pi(R)=\pi(R \cap \sigma(W))=\pi\left(R \cap \sigma^{\prime}(W)\right)=\{w, w s\}$ for some $w \in W$; consequently, $R \cap \sigma(W)=\{\sigma(w), \sigma(w s)\}$, and $\left(\sigma^{\prime} \circ \pi\right)(\sigma(w))=$ $\sigma^{\prime}(w) \in R \cap \sigma^{\prime}(W),\left(\sigma^{\prime} \circ \pi\right)(\sigma(w s))=\sigma^{\prime}(w s) \in R \cap \sigma^{\prime}(W)$.

Recall that $x^{t}$ denotes the shortest element in $\operatorname{Res}(x, t)$ (we use this notation only if $t \in \operatorname{In}(\pi(x)))$. Define inductively $x^{t_{1} \ldots t_{i} t_{i+1}}=\left(x^{t_{1} \ldots t_{i}}\right)^{t_{i+1}}$. 
Notice that if $s t=t s$ then $x^{s t}=x^{t s}$, since both chambers are equal to the shortest element in $\operatorname{Res}(x,\{s, t\})$. We will also use the fact that if $\sigma(w)=x$ for a section $\sigma$ of $\pi$, then $\sigma(w t)=x^{t}$ (assuming $t \in \operatorname{In}(w)$ ).

Lemma 4.8. For any $x \in X$ there exists a morphism $\sigma: W \rightarrow X$ which is a section of $\pi$ and satisfies $\sigma(\pi(x))=x$.

Proof. Induction on the length $k$ of $\pi(x)$. For $k=0$ we have $x=B$, and we just have to show the existence of a section of $\pi$. A morphism $\sigma: W \rightarrow X$ such that $\sigma(1)=B$ can be constructed as in Theorem 4.2. Moreover, since $[x]_{\sim_{s}}$ has at least two elements for each $x \in X, s \in S$, there exists $\sigma$ which is injective on $[x]_{\sim_{s}}$ for each $(x, s) \in R(W)$, hence (by Theorem 4.3(ii)) is a local monomorphism. Then, by Lemma 4.7, $\sigma$ is a section.

Now let $k>0$. Then we can find $w$ of length $k-1$ such that $\pi(x)=w s$ for some $s \in T=\operatorname{In}(w s)$, and a section $\xi: W \rightarrow X$ of $\pi$ such that $\xi(w)=x^{s}$. Let $u s$ be the shortest element in $H(w, s)$, and let $w s, w s t_{1}, \ldots, w s t_{1} \ldots t_{k}=u s$ be a minimal gallery with $t_{i} \in\{s\}^{\prime}$ (cf. Lemma 2.5). We choose a wellordering on $W$ with star-like initial segments such that all elements of $H(w, s)$ are larger than all other elements. If $\{g, g t\} \cap H(w, s)=\emptyset$ and $(g, t) \in R(W)$ then we put $\sigma(g t)=\xi(g t)$ (so that $\sigma$ and $\xi$ coincide on $W \backslash H(w, s))$. Then we put $\sigma(u s)=x^{t_{1} \ldots t_{k}}$, and afterwards we only care about making injective choices, so as to keep $\sigma$ a local monomorphism (and hence, as in the case $k=0$, a section of $\pi$ ).

We claim that $\sigma(w s)=x$. To check this we prove by descending induction on $i$ that $\sigma\left(w s t_{1} \ldots t_{i}\right)=x^{t_{1} \ldots t_{i}}$. Indeed, observe that $\sigma\left(w t_{1} \ldots t_{i}\right)$ $=\xi\left(w t_{1} \ldots t_{i}\right)=x^{s t_{1} \ldots t_{i}}=x^{t_{1} \ldots t_{i} s}$, while by the inductive assumption $\sigma\left(w s t_{1} \ldots t_{i+1}\right)=x^{t_{1} \ldots t_{i+1}}$. Since $x^{t_{1} \ldots t_{i}}$ is the unique chamber which is respectively $s$-and $t_{i+1}$-adjacent to the above two chambers, it has to be equal to $\sigma\left(w s t_{1} \ldots t_{i}\right)$.

For $x \in X$ we can find a standard $W$-building $X^{\prime}$ and a covering map $\phi: X^{\prime} \rightarrow X$ with $\phi\left(B^{\prime}\right)=x$ (Theorem 4.4; recall that a standard $W$-building is a local $W$-building). The corresponding covering map of topological spaces $|\phi|:\left|X^{\prime}\right| \rightarrow|X|$ is a homeomorphism, because, by Lemma 4.6, its base space $|X|$ is contractible, hence simply connected. It follows that $\phi$ itself is an isomorphism. We will now prove that $X$ satisfies the conditions (B1-3). Let $y \in X$, and let $\phi^{-1}(y)=y^{\prime}$. By Lemma 4.8 there exists an apartment $A$ in $X^{\prime}$ containing $B^{\prime}$ and $y^{\prime}$. The $\phi$-image of $A$ is an apartment in $X$ containing $x$ and $y$, which proves (B1). If two apartments $\sigma(W), \sigma^{\prime}(W)$ contain $x$ and $y$, then $\left(\phi^{-1} \circ \sigma\right)(W)$ and $\left(\phi^{-1} \circ \sigma^{\prime}\right)(W)$ contain $B^{\prime}$ and $y^{\prime}$ and thus are isomorphic by an isomorphism $\eta$ fixing $B^{\prime}$ and $y^{\prime}$. Then $\phi \circ \eta \circ \phi^{-1}$ is an isomorphism between $\sigma(W), \sigma^{\prime}(W)$ fixing $x$ and $y$, which proves (B2). The proof of (B3) is analogous to the proof of (B2). - Theorem 4.5 
THEOREM 4.9. For any collection $\left(q_{s}\right)_{s \in S}$ of positive integers there exists a unique $W$-building with $s$-residues of cardinality $q_{s}+1$.

Proof. The construction of the previous subsection with $q_{x, s}=q_{s}$ for all $x$ yields a standard $W$-building $X$ whose residues have required cardinalities. By Theorem 4.5, $X$ is a building. If $Y$ is another building as in the theorem, then as in the proof of Theorem 4.2 one can construct a morphism $\phi: X \rightarrow Y$. Moreover, since the residue cardinalities agree, we can choose $\phi$ so that it is bijective on each $[x]_{\sim_{s}}$ for $(x, s) \in R(X)$. By Theorem $4.3(\mathrm{iv}), \phi$ is a covering map, and hence, by contractibility of $|Y|$, an isomorphism.

One might call the building from Theorem 4.9 a regular $W$-building, with notation $X(W, \mathbf{q})$ (where $\left.\mathbf{q}=\left(q_{s}\right)_{s \in S}\right)$. We will now present another construction of $X(W, \mathbf{q})$. First, we define an auxiliary local building $Y$. The set of chambers of $Y$ is a product $\prod_{s \in S} Y_{s}$, where each $Y_{s}$ is a finite set of cardinality $q_{s}+1$ greater than 1 . Two chambers $\left(y_{s}\right),\left(y_{s}^{\prime}\right)$ are $t$-adjacent if $y_{s}=y_{s}^{\prime}$ for all $s \neq t$. If $T$ is spherical then $\operatorname{Res}_{Y}\left(\left(y_{s}\right), T\right)$ is isomorphic to the product building $\prod_{t \in T} Y_{t}$ (via dropping coordinates indexed by $S \backslash T$ ). Therefore $Y$ is a local $W$-building with the required residue cardinalities, and $\widetilde{Y}=X(W, \mathbf{q})$. It follows that $|X(W, \mathbf{q})|$ carries a free and cocompact action of $\Gamma=\pi_{1}(|Y|)$. If $W$ is a hyperbolic group, then $|X(W, \mathbf{q})|$ is $\operatorname{CAT}(-1)$ and is quasi-isometric to $\Gamma$; therefore, $\Gamma$ is Gromov-hyperbolic (in fact, any group acting cocompactly and properly discontinuously on $|X(W, \mathbf{q})|$ is Gromovhyperbolic).

Proposition 4.10. The building $|X(W, \mathbf{q})|$ carries a free and cocompact action of some group $\Gamma$. If $W$ is hyperbolic, then $\Gamma$ is Gromov-hyperbolic.

Both the proposition and the method of proof (the identification of $X(W, \mathbf{q})$ with the universal cover of $Y)$ are well known (cf. [D2], [GP]).

4.E. Small maps with disjoint images. A (standard or local) $W$ building is thick if each adjacency class has at least three elements.

THEOREM 4.11. Let $X$ be a thick standard $W$-building, and let $N \subseteq X$ be a finite set. Then there exists a finite set $M$ with $N \subseteq M \subseteq X$ and two $\pi$-equivariant maps $\phi, \psi: X \rightarrow X$ such that $\left.\phi\right|_{M}=\left.\psi\right|_{M}=\operatorname{Id}_{M}$ and $\phi(X \backslash M) \cap \psi(X \backslash M)=\emptyset$.

Proof. Put $M=\pi^{-1}(\operatorname{conv}(\pi(N) \cup\{1\}))$. We claim that $\operatorname{conv}(\pi(N) \cup\{1\})$, hence $M$, is finite. Indeed, let $m$ be the number of walls in $W$ separating some element of $\pi(N)$ from 1 . Let $\ell(w)=p>m$, and let $w_{0}=1, w_{1}, \ldots, w_{p}=w$ be a minimal gallery. Then one of the $p$ walls between $w_{i}$ and $w_{i+1}$ does not separate any element of $\pi(N)$ from 1 , while it separates $w$ from 1 ; hence, it separates $w$ from $\pi(N) \cup\{1\}$, and $w \notin \operatorname{conv}(N \cup\{1\})$. 
Now let $R=R(X)$ be the root set of $X$. For each $(x, s) \in R$ choose two distinct elements $a_{(x, s)}, b_{(x, s)} \in \operatorname{Res}(x, s) \backslash\{x\}$. Let $A=\left\{a_{r} \mid r \in R\right\}, B=$ $\left\{b_{r} \mid r \in R\right\}$. We now construct $\phi: X \rightarrow X$ as in the proof of Theorem 4.2. Let $x \in X$ be such that $\operatorname{In}(\pi(x))=\{s\}$ (i.e., we have a choice for $\phi(x))$. Let $x_{0}$ be the shortest element of $\operatorname{Res}(x, s)$; then $\left(x_{0}, s\right) \in R$. If $x \in M$ we put $\phi(x)=x$ (this is allowed, for by induction $\phi\left(x_{0}\right)=x_{0}$ ). If $x \notin M$, we put $\phi(x)=a_{\left(\phi\left(x_{0}\right), s\right)}$. Similarly we define $\psi$ using $b$ 's instead of $a$ 's.

Suppose now that $y \in \phi(X) \cap \psi(X), y \notin M$, and $w=\pi(y)$ is the shortest possible. Let $y=\phi(x)=\psi(z)$. We have $\phi\left(x^{t}\right)=\psi\left(z^{t}\right)$ for all $t \in \operatorname{In}(w)$, hence $x^{t}=z^{t} \in M$ for all such $t$. Now $y \notin M$ is possible only if $\operatorname{In}(w)$ has one element, say $t$. But then $\left(x^{t}, t\right) \in R,\left(z^{t}, t\right) \in R, \phi(x) \in A, \psi(z) \in B$, a contradiction.

Let

$$
|M|=\left\{[x, p] \mid x \in M, p \in C L^{\prime}\right\}, \quad|\phi|([x, p])=[\phi(x), p] .
$$

Corollary 4.12. Let $\phi, \psi$ be as in Theorem 4.11. Then

$$
|\phi|(|X| \backslash|M|) \cap|\psi|(|X| \backslash|M|)=\emptyset .
$$

Proof. Suppose not; let $[x, p]=|\phi|\left(\left[x_{1}, p\right]\right)=|\psi|\left(\left[x_{2}, p\right]\right)$. Recall that $S(p)=\left\{s \in S\left|\exists \sigma \in L^{\prime}, s \in \sigma, p \in\right| \sigma \mid\right\}$. Let $y$ be the shortest element of $\operatorname{Res}(x, S(p))$, and let $y_{1}$ be the shortest element of $\operatorname{Res}\left(x_{1}, S(p)\right)$; then $[x, p]=[y, p]$ and $\left[x_{1}, p\right]=\left[y_{1}, p\right]$. Since $\operatorname{Res}(x, S(p))$ is the unique residue $R$ of type $S(p)$ in $X$ such that $\left|X_{\leq R}\right|$ contains $[x, p]$ (for two different residues $R$ of the same type the sets $\left|X_{\leq R}\right|$ are disjoint $)$, we have $|\phi|\left(\left|X_{\leq \operatorname{Res}\left(x_{1}, S(p)\right)}\right|\right) \subseteq$ $\left|X_{\leq \operatorname{Res}(x, S(p))}\right|$, and hence $\phi\left(\operatorname{Res}\left(x_{1}, S(p)\right)\right) \subseteq \operatorname{Res}(x, S(p))$. Now $\pi$-equivariance of $\phi$ implies that $\phi\left(y_{1}\right)=y$. Similarly, $\psi\left(y_{2}\right)=y$. It follows that $y \in M$ and $[x, p]=[y, p] \in|M|$.

If $X$ is a right-angled hyperbolic building with a folding map $\pi$, then any $\pi$-equivariant map $\theta: X \rightarrow X$ fixes the base chamber $B=\pi^{-1}(1)$. Therefore $|\theta|:|X| \rightarrow|X|$ fixes all points in $B$. Recall that we defined $\partial|X|$ as the space of geodesic rays starting at some base point $x_{0} \in B$. Thus, the map $|\theta|$ induces a continuous map $\partial|\theta|: \partial|X| \rightarrow \partial|X|$.

COROLlaRY 4.13. Let $X$ be a right-angled hyperbolic building, and let $\phi, \psi$ be as in Theorem 4.11. Then

$$
\partial|\phi|(\partial|X|) \cap \partial|\psi|(\partial|X|)=\emptyset .
$$

Proof. Suppose not; let $\partial|X| \ni z=\partial|\phi|\left(z_{1}\right)=\partial|\psi|\left(z_{2}\right)$. For $y \in \partial|X|$ let $\gamma_{y}:[0, \infty) \rightarrow|X|$ be the geodesic from the base point to $y$. We have $\gamma_{z}=$ $|\phi| \circ \gamma_{z_{1}}=|\psi| \circ \gamma_{z_{2}}$. Let $t \in[0, \infty)$ be so large that $\gamma_{z_{1}}(t), \gamma_{z_{2}}(t) \notin|M| ;$ then $\gamma_{z}(t)=|\phi|\left(\gamma_{z_{1}}(t)\right)=|\psi|\left(\gamma_{z_{2}}(t)\right)$, contradicting Corollary 4.12. 


\section{APPENDIX}

In this appendix we prove an analogue of Lemma 2.15 for arbitrary finite spherical buildings (Theorem A.2). As a corollary, we deduce that an $n$-dimensional locally finite hyperbolic or Euclidean building (not necessarily right-angled) is $(n-2)$-connected at infinity. Note that in [GP] even more is claimed, but their proof does not convince us. First, it is not true that $V \cap S\left(x, s_{i}+\varepsilon\right)$ (here we refer to the proof of Proposition 4.1 in [GP] and we use the notation used there) is of the same homotopy type as the pointed connected sum of $S\left(x, s_{i}-\varepsilon\right)$ with a bouquet of spheres-one for each chamber opposite to $c$ in $L k(y)$. This can be seen by considering a 2-dimensional right-angled building. Second, to claim that $V \cap S\left(x, s_{i}+\varepsilon\right)$ has the homotopy type of a bouquet of spheres, one needs to show that $S_{+}$is $(n-2)$-connected (in the notation of [GP]). This is, in our opinion, a non-trivial fact - see Theorem A.2 below. A similar problem appears in [DM]. Again, as consideration of a 2-dimensional right-angled building shows, Lemma 5.5 in [DM] is false (cf. $\left.\left[\mathrm{DM}^{\prime}\right]\right)$. We do not know how to correct this approach.

Kai-Uwe Bux informs us that variants of Theorem A.2 and Corollary A.10 have been independently established by B. Schulz in his thesis (cf. [Sch]).

Let $X$ be a finite spherical building of dimension $n \geq 1$, equipped with the standard CAT(1) metric (each apartment is a sphere of diameter $\pi$ ). Let $B \in X$ be a chamber, and let $\pi: X \rightarrow S^{n}$ be the $B$-based folding map. We equip $S^{n}$ with the standard round metric such that the restriction of $\pi$ to any apartment $A$ containing $B$ isometrically identifies $A$ and $S^{n}$. The triangulation of $A$ transported by $\pi$ is a triangulation of $S^{n} ; \pi: X \rightarrow S^{n}$ is then a simplicial map. Images of chambers under $\pi$ will be called chambers.

LemmA A.1. Let $S_{1}, \ldots, S_{k} \subseteq S^{n}$ be a finite collection of great spheres (of arbitrary dimensions). The set of points $x$ satisfying:

- for every $i$, the function $S_{i} \ni y \mapsto d(x, y) \in \mathbb{R}$ has a unique minimum;

- for every $i \neq j, d\left(x, S_{i}\right) \neq d\left(x, S_{j}\right)$;

is open and dense in $S^{n}$.

For every simplex $\sigma$ in our triangulation of $S^{n}$ there exists a unique smallest great sphere $S \subseteq S^{n}$ containing $\sigma$. Apply Lemma A.1 to the collection of all spheres thus obtained; pick a point $\bar{x} \in S^{n}$ in the dense open set given by the lemma and inside $\operatorname{int}(\pi(B))$. Let $E^{0}=\bar{x}^{\perp} \cap S^{n}$ be the equator of $S^{n}$ for which $\bar{x}$ is a pole; let $E^{-}$be the closed hemisphere with boundary $E^{0}$ containing $\bar{x}$, and let $E^{+}$be the other closed hemisphere with boundary $E^{0}$. Also, let

$$
X^{0}=\pi^{-1}\left(E^{0}\right), \quad X^{-}=\pi^{-1}\left(E^{-}\right), \quad X^{+}=\pi^{-1}\left(E^{+}\right) .
$$


For a subset $U$ or point $p$ of $X$ we put

$$
\bar{U}=\pi(U), \quad \bar{p}=\pi(p) .
$$

We will often pick $\bar{U}$ or $\bar{p}$ first, and specify $U$ or $p$ later (if at all). For example, $x$ is the unique point in $\pi^{-1}(\bar{x})$. To avoid a notational clash, closures will be denoted by $\mathrm{cl}$.

TheOREM A.2. Let $X$ be a finite spherical building of dimension $n \geq 1$. Then $X^{+}$is $(n-1)$-connected.

Proof. If a set $G \subseteq X$ is isomorphically mapped by $\pi$ onto $H \subseteq S^{n}$, we say that $G$ folds onto $H$. The following lemma will often be used:

LEMMA A.3.

(a) For every chamber $C \in X$ there exists an apartment $A$ containing $C$ such that $A$ folds onto $S^{n}$.

(b) Let $C_{1}, C_{2}$ be two chambers in $X$ such that $\pi\left(C_{2}\right)=-\pi\left(C_{1}\right)$. Then there exists a unique apartment $A$ containing $C_{1}$ and $C_{2}$. This $A$ folds onto $S^{n}$.

Proof. (a) An apartment containing $C$ and $B$ is good. (b) $C_{1}, C_{2}$ are opposite in $X$-otherwise $\bar{C}_{1}, \bar{C}_{2}$ would not be opposite in $S^{n}$. Consequently, $\operatorname{conv}\left(C_{1} \cup C_{2}\right)$ is the desired apartment.

REMARK. One can replace the chamber $C$ in part (a) by a point; similarly, one can replace chambers $C_{1}, C_{2}$ in part (b) by points $p, q$ such that $\bar{q}=-\bar{p}$ and $p$ is in the interior of some chamber. (Choose chamber/pair of opposite chambers containing the point/points, and apply the lemma.)

LemmA A.4. Let $n \geq 1$. Then $X^{+}$is path-connected.

Proof. Let $p, q \in X^{+}$. Choose apartments $A_{p}, A_{q}$ that fold onto $S^{n}$ and contain $p, q$, respectively. Pick a point $p^{\prime} \in X^{0} \cap A_{p}$ which lies in the interior of some chamber $C_{p}$ (this is possible due to genericity of $\bar{x}$ ). Let $q^{\prime} \in X^{0} \cap A_{q}$ be such that $\overline{q^{\prime}}=-\overline{p^{\prime}}$; let $C_{q}$ be the chamber which contains $q^{\prime}$. Then $p$ can be connected to $p^{\prime}$ by a path in $X^{+} \cap A_{p}$, and $q$ can be connected to $q^{\prime}$ by a path in $X^{+} \cap A_{q}$. Furthermore, $\pi\left(C_{q}\right)=-\pi\left(C_{p}\right)$ so that, by Lemma A.3, there exists an apartment $A \ni C_{p}, C_{q}$ which folds onto $S^{n}$. Now $p^{\prime}$ and $q^{\prime}$ can be connected by a path in $X^{+} \cap A$. Thus, $p$ and $q$ can be connected by a path in $X^{+}$.

\section{Proposition A.5. Let $n=2$. Then $\pi_{1}\left(X^{+}\right)=0$.}

Proof. By a general position argument, any loop in $X^{+}$can be homotoped to a loop in $X^{+} \backslash \pi^{-1}(-\bar{x})$. The latter set deformation retracts onto $X^{0}$ (the deformation retraction moves a point along the unique shortest geodesic towards $x$, until it hits $\left.X^{0}\right)$. Consequently, any loop in $X^{+}$can be homotoped to a loop in $X^{0}$. Now $X^{0}$ has a natural graph structure, inherited from the 
simplicial structure of $X$. Therefore, a loop in $X^{0}$ is homotopic to a simplicial loop $\sigma=\left(e_{1}, \ldots, e_{k}=e_{0}\right.$ ) (each $e_{i}$ is an oriented edge and the endpoint of $e_{i}$ is the origin of $\left.e_{i+1}\right)$. A pair $\left(e_{i}, e_{i+1}\right)$ will be called a backtracking pair (b.p.) if $\bar{e}_{i}=\bar{e}_{i+1}^{-}$(we use $f^{-}$to denote $f$ with reversed orientation). Now choose an edge $\bar{e}$ in $E^{0}$. A b.p. $\left(e_{i}, e_{i+1}\right)$ is called acceptable if $\bar{e}_{i}= \pm \bar{e}^{ \pm}$(one of the four possibilities). If $\left(e_{i}, e_{i+1}\right)$ is a b.p., we choose an apartment $A$ that contains $e_{i}$ and folds onto $S^{n}$. Then $A \cap X^{0}$ is a loop $\left(e_{i}, f_{2}, f_{3}, \ldots, f_{2 s}\right)$. There exists a smallest $j \geq 2$ such that $\bar{f}_{j}= \pm \bar{e}^{ \pm}$; we deform the loop $\sigma=\left(\ldots, e_{i}, e_{i+1}, \ldots\right)$ to $\sigma^{\prime}=\left(\ldots, e_{i}, f_{2}, \ldots, f_{j}, f_{j}^{-}, \ldots, f_{2}^{-}, e_{i+1}, \ldots\right)$. The new loop has the same backtracking pairs as $\sigma$, with the exception of $\left(e_{i}, e_{i+1}\right)$, instead of which an acceptable b.p. $\left(f_{j}, f_{j}^{-}\right)$appears. Notice that the b.p. $\left(f_{j}, f_{j}^{-}\right)$is separated, in the sense that neither $\left(f_{j-1}, f_{j}\right)$ nor $\left(f_{j}^{-}, f_{j-1}^{-}\right)$is a b.p. (if $j=2$, neither $\left(e_{i}, f_{2}\right)$ nor $\left(f_{2}^{-}, e_{i+1}\right)$ is a b.p.). Repeating the process, we deform $\sigma$ to a loop with acceptable separated backtracking pairs only. We keep the notation $\sigma=\left(e_{1}, \ldots, e_{k}=e_{0}\right)$ for this new loop.

Now suppose that $\bar{e}_{i}= \pm \bar{e}^{ \pm}$, but neither $\left(e_{i}, e_{i+1}\right)$ nor $\left(e_{i-1}, e_{i}\right)$ is a b.p. Then $\bar{e}_{i+s}=-\bar{e}_{i}$ so that, by Lemma A.3 and the subsequent remark, there exists an apartment $A \ni e_{i}, e_{i+s}$. We claim that $e_{i+1}, \ldots, e_{i+s-1} \in A$.

Lemma A.6. Let $\tau=\left(d_{1}, \ldots, d_{s+1}\right)$ be a path in $X^{0}$ such that $\bar{d}_{1}=$ $-\bar{d}_{s+1}$, and let $A$ be the apartment in $X$ containing $d_{1}$ and $d_{s+1}$. Then $\tau$ is contained in $A$.

Proof. The path $\tau^{\prime}=\left(d_{2}, \ldots, d_{s}\right)$ from the endpoint $y$ of $d_{1}$ to the origin $z$ of $d_{s+1}$ has geometric length $d(\bar{y}, \bar{z})$. Since $d(\bar{y}, \bar{z}) \leq d(y, z), \tau^{\prime}$ is a shortest geodesic. Now $y, z \in A$, and $A$ is convex, therefore $\tau$ is contained in $A$.

In $A \cap X^{+}$, the path $\left(e_{i+1}, e_{i+2}, \ldots, e_{i+s-1}\right)$ is homotopic (with endpoints fixed) to a path $\left(e_{i}^{-}, f_{i+1}, \ldots, f_{i+s-1}, e_{i+s}^{-}\right)$, where $\bar{f}_{i+j}=-\bar{e}_{i+s-j}^{-}$. The effect of this change on $\sigma$ is

$$
\begin{aligned}
\left(\ldots, e_{i}, e_{i+1}, \ldots, e_{i+s-1}, e_{i+s}, \ldots\right) & \\
& \rightarrow\left(\ldots, e_{i}, e_{i}^{-}, f_{i+2}, \ldots, f_{i+s-1}, e_{i+s}^{-}, e_{i+s}, \ldots\right) .
\end{aligned}
$$

It may happen that $\left(e_{i+s}, e_{i+s+1}\right)$ is a b.p.; if this is the case, we further modify the loop:

$$
\left(\ldots, f_{i+s-1}, e_{i+s}^{-}, e_{i+s}, e_{i+s+1}, \ldots\right) \rightarrow\left(\ldots, f_{i+s-1}, e_{i+s+1}, \ldots\right) .
$$

Travelling along the loop and repeating the process if necessary, we finally arrive at a loop $\sigma=\sigma_{1} \ldots \sigma_{2 l}$, where each $\sigma_{i}$ is a path of length $s+1$ with no b.p., and (last edge of $\sigma_{i}$, first edge of $\sigma_{i+1}$ ) is an acceptable separated b.p. (for $i=0,1, \ldots, 2 l-1$, where $\sigma_{0}=\sigma_{2 l}$ ).

Suppose now that $\tau$ is a path of length $s+1$ containing no b.p. Let $A$ be the apartment containing the extreme edges of $\tau$. Then $\tau$ is homotopic (with 
endpoints fixed) in $A \cap X^{+}$to a path $\widehat{\tau} \subseteq A \cap X^{0}$ of length $s-1$. Now we modify $\sigma$ by homotopy inside $X^{+}$, changing $\sigma_{j}$ and $\sigma_{2 l-j+1}$ to $\widehat{\sigma}_{j}, \widehat{\sigma}_{2 l-j+1}$ (resp.), for all positive even $j \leq l$. We obtain a loop $\sigma=\eta \xi$ with exactly two backtracking pairs, where $\eta, \xi$ are paths of equal length, say $u$, and none of them contains a b.p. A loop of this form will be called a $u$-moon.

Lemma A.7. An $(s+1)$-moon is contractible in $X^{+}$.

Proof. An $(s+1)$-moon $\eta \xi$ is homotopic (in $\left.X^{+}\right)$to $\eta \widehat{\xi}$; the latter is contained, by Lemma A.6, in the apartment $A$ spanned by the extreme edges of $\eta$. The apartment $A$ folds onto $S^{n}$, therefore $\eta \widehat{\xi}$ is null-homotopic in $A \cap X^{+}$.

Lemma A.8. If $u>s+1$, then a $u$-moon is homotopic to a concatenation of an $(s+1)$-moon and $a(u-1)$-moon.

Proof. Let $\eta=\left(\eta_{1}, \ldots, \eta_{u}\right)$ and $\xi=\left(\xi_{1}, \ldots, \xi_{u}\right)$. Let $A$ be the apartment spanned by $\eta_{1}$ and $\xi_{u-s}$, and let $\tau$ be the path of length $s-1$ in $A \cap X^{0}$ from the endpoint of $\eta_{1}$ to the endpoint of $\xi_{u-s}$. Then $\eta \xi$ is homotopic to the concatenation of the $(s+1)$-moon $\tau \xi_{u-s}^{-} \xi_{u-s} \xi_{u-s+1} \ldots \xi_{u} \eta_{1}$ and the $(u-1)$-moon $\eta_{2} \eta_{3} \ldots \eta_{u} \xi_{1} \xi_{2} \ldots \xi_{u-s} \tau^{-}$. $\cdot$ Lemma A.8

Repeated application of Lemmas A.8 and A.7 finishes the proof of Proposition A.5. Proposition A.5

Thus, the assertion of Theorem A.2 is true for $n=1,2$. We will proceed with the proof of the general case by induction on $n$. Suppose that $n>2$, and that the assertion of Theorem A.2 is true for all finite buildings of dimension less than $n$. Let $X$ be a finite building of dimension $n$.

Let $\sigma_{1}, \ldots, \sigma_{l}$ be all the simplices of our triangulation of $S^{n}$ that have the following property: there exists a minimal unit-speed geodesic $\bar{\gamma}_{i}$ issued from $\bar{x}$ which orthogonally intersects the interior of $\sigma_{i}$ at $\bar{p}_{i}=\bar{\gamma}_{i}\left(t_{i}\right)$. By the choice of $\bar{x}, \bar{\gamma}_{i}$ is unique, all the $t_{i}$ are distinct and none of them equals $\pi / 2$. We can assume that $t_{1}<\cdots<t_{l}$. Let

$$
X_{r}^{+}=\{y \in X \mid d(\pi(y), \bar{x}) \geq r\} .
$$

Our strategy is to show, by induction on $i$, that $X_{r}^{+}$is $(n-1)$-connected for $t_{i}<r<t_{i+1}, r \leq \pi / 2$. To this end, we need to prove that $X_{\varepsilon}^{+}$is $(n-1)$-connected for small positive $\varepsilon$, and then we need to understand how $X_{r}^{+}$changes when $r$ switches from the interval $\left(t_{i-1}, t_{i}\right)$ to $\left(t_{i}, t_{i+1}\right)$.

If $\varepsilon$ is sufficiently close to 0 , then $X_{\varepsilon}^{+}$is homotopy equivalent to $X \backslash\{x\}$, which homotopically is a bouquet of $n$-spheres with one sphere punctured, and so is $(n-1)$-connected.

Now we will closely follow the proof of Lemma 3.3. Put $t=t_{i}, \sigma=\sigma_{i}$, $\bar{\gamma}=\bar{\gamma}_{i}, \bar{p}=\bar{p}_{i}$. We choose a small $\delta>0$ such that the sphere $S_{\delta}(\bar{p})$ is contained in $\operatorname{int}(\operatorname{Res} \sigma)$, where $\operatorname{Res} \sigma=\bigcup\{\tau \mid \sigma \subseteq \tau\}$. Then there exists 
an $\varepsilon>0$ such that $S_{t+\varepsilon}(\bar{x}) \cap \sigma \subseteq S_{\delta}(\bar{p})$. The intersection $S_{t+\varepsilon}(\bar{x}) \cap \sigma$ is a sphere of dimension $d=\operatorname{dim} \sigma-1$ contained in the interior of $\sigma$. Decreasing $\delta$ we ensure that $\varepsilon<\min \left\{t_{i+1}-t, t-t_{i-1}\right\}$ and $t+\varepsilon<\pi / 2$. Let $\bar{D}$ denote $S_{\delta}(\bar{p}) \cap \sigma_{\bar{p}}^{\perp}$ (where $\eta_{\bar{p}}^{\perp}$ denotes the largest great sphere in $S^{n}$ orthogonal to $\eta$ at $\bar{p}$ ). The intersection $\bar{D} \cap \bar{\gamma}_{\bar{p}}^{\perp}$ divides $\bar{D}$ into two closed hemispheres: $\bar{D}^{-}$(the one closer to $\bar{x}$ ) and $\bar{D}^{+}$. Observe that $\bar{D}^{+}=\bar{D} \backslash B_{t+\varepsilon}(\bar{x})$. The sphere $\bar{D}$ inherits a triangulation from $S^{n}$. We want the spheres $S_{t \pm \varepsilon}(\bar{x})$ to intersect this triangulation "in the same way". This condition can be achieved by further decreasing $\delta$ (and consequently $\varepsilon$ ).

Now we pass to $X$. Let

$$
\begin{gathered}
\pi^{-1}(\sigma)=\left\{\sigma_{1}, \ldots, \sigma_{k}\right\}, \quad\left\{p_{s}\right\}=\sigma_{s} \cap \pi^{-1}(\bar{p}), \\
K_{s}=\left\{y \in X \mid d\left(y, p_{s}\right) \leq \delta\right\}, \quad K=\coprod_{s} K_{s}, \quad Y^{+}=\operatorname{cl}\left(X_{t+\varepsilon}^{+} \backslash K\right), \\
D_{s}=\pi^{-1}(\bar{D}) \cap K_{s}, \quad D_{s}^{+}=D_{s} \cap X_{t+\varepsilon}^{+}, \quad S_{s}^{d}=\sigma_{s} \cap S_{\delta}\left(p_{s}\right) .
\end{gathered}
$$

The first two definitions override our previous convention. Observe that: $X_{t+\varepsilon}^{+}$ is homotopy equivalent to $Y^{+} ; X_{t-\varepsilon}^{+}$is homotopy equivalent to $Y^{+} \cup K ; K_{s}$ is homeomorphic to a cone over the join $S_{s}^{d} * D_{s}$, and is attached to $Y^{+}$along a subset homeomorphic to $S_{s}^{d} * D_{s}^{+}$.

Each $D_{s}$ is a spherical building of dimension $n-d-2$. By the inductive assumption $D_{s}^{+}$is $(n-d-3)$-connected, which implies that $S_{s}^{d} * D_{s}^{+}$is $(n-2)$ connected. Moreover, $K_{s}$ is contractible. Van Kampen's theorem, MayerVietoris sequence and the inductive assumption that the union $Y^{+} \cup K$ is $(n-1)$-connected imply $(n-2)$-connectedness of $Y^{+}$. It remains to prove that $H_{n-1}\left(Y^{+}\right)=0$ (here we depart from the proof of Lemma 3.3). It follows from the Mayer-Vietoris sequence that $H_{n-1}\left(Y^{+}\right)$is generated by the images of $H_{n-1}\left(S_{s}^{d} * D_{s}^{+}\right)(s=1, \ldots, k)$. We will show that any $(n-1)$-cycle in $S_{s}^{d} * D_{s}^{+}$ is null-homologous in $Y^{+}$.

Let us subdivide the usual triangulation of $S^{n}$ to a minimal cellulation in which $S_{t+\varepsilon}(\bar{x}) \backslash B_{\delta}(\bar{p})$ and $S_{\delta}(\bar{p})$ are subcomplexes. Pull this cellulation back to $X$ via $\pi$. An $(n-1)$-cycle $z$ in $S_{s}^{d} * D_{s}^{+}$is a join of the fundamental class of $S_{s}^{d}$ and an $(n-d-2)$-cycle $\widetilde{z}$ in $D_{s}^{+}$. The cycle $\widetilde{z}$ can be regarded as a cycle in $D_{s}$ vanishing outside $D_{s}^{+}$. Now every $(n-d-2)$-cycle in $D_{s}$ can be expressed as a combination of fundamental classes of apartments. More specifically, let $c_{0}$ be the chamber in $D_{s}$ which is closest to $x$, and let $c_{1}, \ldots, c_{m}$ be all the chambers in $D_{s}$ opposite to $c_{0}$. Let $a_{i}$ be the apartment in $D_{s}$ containing $c_{0}$ and $c_{i}$; then $\widetilde{z}=\sum_{i=1}^{m} \alpha_{i}\left[a_{i}\right]$ for some integers $\alpha_{i}$. Let $C_{i}$ be the chamber of $X$ containing $c_{i}$. Let $C_{-1}$ be a chamber in $X$ such that $\bar{C}_{-1}=-\bar{C}_{1}$ (note that $\bar{C}_{1}=\cdots=\bar{C}_{m}$ ), and let $A_{i}$ be the apartment in $X$ containing $C_{-1}$ and $C_{i}$. Let $Z=\sum_{i=0}^{m} \alpha_{i}\left[A_{i}\right] \in Z_{n}(X)$. Split $Z$ into $Z_{1}+Z_{2}$, 
where $Z_{1} \in C_{n}\left(K_{s}\right), Z_{2} \in C_{n}\left(\operatorname{cl}\left(X \backslash K_{s}\right)\right)$. Clearly, $Z_{1}$ is the cone over $z$ so that $\partial Z_{1}=z$. Therefore, $\partial\left(-Z_{2}\right)=z$.

We claim that $Z_{2} \in C_{n}\left(Y^{+}\right)$. First, notice that if $u \neq s$ then for all $i$ we have $K_{u} \cap A_{i}=\emptyset$ : since $A_{i}$ folds onto $S^{n}$, it can intersect only one component of $K$, and it does intersect $K_{s}$. Next, let $C$ be a chamber of $X$ contained in $A_{i}$ but not contained in $Y^{+} \cup K$. Choose a point $y \in \operatorname{int}(C)$ such that $y \notin X_{t}^{+} \cup K$ (in particular, $\bar{y} \neq-\bar{p}$ ). The unique minimal geodesic $\gamma$ from $p_{s}$ to $y$ is contained in $\left(A_{i} \cap\left(X \backslash X_{t}^{+}\right)\right) \cup\left\{p_{s}\right\}$, because both extremities belong to this convex set. In particular, $\gamma$ intersects $S_{\delta}\left(p_{s}\right)$ outside $X_{t}^{+}$, hence outside $Y^{+}$. It follows that $\gamma$ leaves $p_{s}$ through the interior of a chamber $C^{\prime}$ on which $Z$ is zero. The chamber $C^{\prime}$ is the closest to $C$ (in the gallery distance) among all chambers in the residue of $\sigma$.

Now all apartments $A_{j}$ contain $\sigma$; therefore, if an apartment $A_{j}$ contains $C$, it also contains $C^{\prime}$. Conversely, we claim that if $C^{\prime} \in A_{j}$, then $C \in A_{j}$. To see this extend the geodesic $\gamma$ to $\gamma:[0, \pi] \rightarrow A_{i}$ (so that $\gamma(\pi)$ is opposite to $\gamma(0)=p_{s}$ in $\left.A_{i}\right)$. Now slightly rotate $\gamma$ inside $A_{i}$, so as to obtain a geodesic $\eta$ which still passes through the interiors of $C^{\prime}$ and $C\left(\eta(a) \in \operatorname{int}\left(C^{\prime}\right), \eta(b) \in\right.$ $\operatorname{int}(C), a<b)$, but $\eta(0) \in \operatorname{int}\left(C_{i}\right), \eta(\pi) \in \operatorname{int}\left(C_{-1}\right)$. Then $\left.\eta\right|_{[a, \pi]}$ is a minimal geodesic. Suppose that $C^{\prime} \in A_{j}$. Then $\eta(a), \eta(\pi) \in A_{j}$ so that $\eta(b) \in A_{j}$, hence $C \in A_{j}$.

Thus, we have verified that $\left\{j \mid C \in A_{j}\right\}=\left\{j \mid C^{\prime} \in A_{j}\right\}$. The coefficient of $C$ in $Z$ is equal to $\sum_{j \mid C \in A_{j}} \alpha_{j}=\sum_{j \mid C^{\prime} \in A_{j}} \alpha_{j}$; the latter is the coefficient of $C^{\prime}$ in $Z$, i.e. zero. Theorem A.2

A non-compact space $X$ is $k$-connected at infinity if for every compact $K \subseteq X$ there exists a compact set $L$ with $K \subseteq L \subseteq X$ such that any map $S^{i} \rightarrow X \backslash L$ extends to a map $B^{i+1} \rightarrow X \backslash K$ (for $i=0,1, \ldots, k$ ).

COROllary A.9. An n-dimensional locally finite hyperbolic or Euclidean building is $(n-2)$-connected at infinity.

Proof. It is enough to check that the complements of balls $B_{r}$ are $(n-2)$ connected, for $r>0$ arbitrarily large. By the geodesic retraction such a complement is homotopically equivalent to $S_{r}$. Then the proof of Lemma 3.3, for $U=X \cup \partial X$, goes through, with Theorem A.2 used instead of Lemma 2.15. Lemma 2.16 is never needed for this choice of $U$.

Finally, let us remark that in Theorem A.2 the assumptions on $\bar{x}$ can be relaxed.

Corollary A.10. The conclusion of Theorem A.2 is true for all $\bar{x}$ in the closed chamber $\pi(B)$.

Proof. For any $\bar{x} \in \pi(B)$ and any positive $\varepsilon$ one can find a generic $\varepsilon$ approximation of $\bar{x}$ : a point $\bar{x}_{\varepsilon}$ in $\operatorname{int}(\pi(B))$, belonging to the dense open set 
given by Lemma A.1, $\varepsilon$-close to $\bar{x}$. Then

$$
V_{\varepsilon}^{+}:=\left\{y \in X \mid d\left(\pi(y), \bar{x}_{\varepsilon}\right) \geq \pi / 2-\varepsilon\right\}
$$

contains $X^{+}$and is contained in the $\varepsilon$-neighbourhood of $X^{+}$. Since $X^{+}$has a (spherical) finite polyhedral structure, it is an ANR; hence, for $\varepsilon$ small enough, $V_{\varepsilon}^{+}$retracts onto $X^{+}$. By the proof of Theorem A.2, $V_{\varepsilon}^{+}$is $(n-1)$ connected. Therefore, its retract $X^{+}$is also $(n-1)$-connected.

\section{References}

[B] N. Benakli, Polyèdres hyperboliques, passage du local au global, Thèse, Univ. Paris Sud, 1992.

[BK] N. Benakli and I. Kapovich, Boundaries of hyperbolic groups, in: Combinatorial and Geometric Group Theory (New York, 2000/Hoboken, NJ, 2001), Contemp. Math. 296, Amer. Math. Soc., Providence, RI, 2002, 39-93.

[Be] M. Bestvina, Characterizing k-dimensional universal Menger compacta, Mem. Amer. Math. Soc. 71 (1988), no. 380.

[BB] M. Bestvina and N. Brady, Morse theory and finiteness properties of groups, Invent. Math. 129 (1997), 445-470.

[BH] M. Bridson and A. Haefliger, Metric Spaces of Non-Positive Curvature, Springer, 1999.

[Bd1] M. Bourdon, Immeubles hyperboliques, dimension conforme et rigidité de Mostow, Geom. Funct. Anal. 7 (1997), 245-268.

[Bd2] - Sur les immeubles fuchsiens et leur type de quasi-isométrie, Ergodic Theory Dynam. Systems 20 (2000), 343-364.

[BP1] M. Bourdon and H. Pajot, Rigidity of quasi-isometries for some hyperbolic buildings, Comment. Math. Helv. 75 (2000), 701-736.

[BP2] M. Bourdon et H. Pajot, Cohomologie $L^{p}$ et espaces de Besov, J. Reine Angew. Math. 558 (2003), 85-108.

[BCM] N. Brady, J. McCammond and J. Meier, Local-to-asymptotic topology for cocompact CAT(0) complexes, Topology Appl. 131 (2003), 177-188.

[Br] K. Brown, Buildings, Springer, New York, 1989.

[D1] M. W. Davis, Groups generated by reflections and aspherical manifolds not covered by Euclidean space, Ann. of Math. (2) 117 (1983), 293-324.

[D2] M. W. Davis, Buildings are CAT(0), in: Geometry and Cohomology in Group Theory, P. Kropholler et al. (eds.), London Math. Soc. Lecture Note Ser. 252, Cambridge Univ. Press, Cambridge, 1998, 108-123.

[DM] M. W. Davis and J. Meier, The topology at infinity of Coxeter groups and buildings, Comment. Math. Helv. 77 (2002), 746-766.

$\left[\mathrm{DM}^{\prime}\right]-,-$, Erratum to: "The topology at infinity of Coxeter groups and buildings", ibid. 82 (2007), 235-236.

[GP] D. Gaboriau et F. Paulin, Sur les immeubles hyperboliques, Geom. Dedicata 88 (2001), 153-197.

[Gl] M. Globus, unpublished, 1996.

[HP] F. Haglund et F. Paulin, Constructions arborescentes d'immeubles, Math. Ann. 325 (2003), 137-164.

[KK] M. Kapovich and B. Kleiner, Hyperbolic groups with low-dimensional boundary, Ann. Sci. École Norm. Sup. (4) 33 (2000), 647-669. 
[M] G. Moussong, Hyperbolic Coxeter groups, PhD thesis, Ohio State Univ., 1987.

[Ron] M. Ronan, Lectures on Buildings, Perspectives in Math. 7, Academic Press, 1989.

[Sch] B. Schulz, Sphärische Unterkomplexe sphärischer Gebäude, PhD thesis, Johann Wolfgang Goethe-Univ., Frankfurt a.M., 2005.

[Se] J.-P. Serre, Cohomologie des groupes discrets, in: Prospects in Mathematics, Ann. of Math. Stud. 70, Princeton Univ. Press, Princeton, NJ, 1971, 77-169.

[Vin] E. B. Vinberg, Hyperbolic reflection groups, Uspekhi Mat. Nauk 40 (1985), no. 1, 29-66 (in Russian).

Instytut Matematyczny

Uniwersytet Wrocławski

Pl. Grunwaldzki 2/4

50-384 Wrocław, Poland

E-mail: dymara@math.uni.wroc.pl

dosaj@math.uni.wroc.pl
Institut de Mathématiques de Jussieu Université Paris 6 Case 247, 4 Place Jussieu 75252 Paris Cedex 05, France

Received 5 October 2006;

in revised form 23 October 2007 